\title{
Mixed Finite Element Methods
}

\author{
Ricardo G. Durán*
}

\section{Introduction}

Finite element methods in which two spaces are used to approximate two different variables receive the general denomination of mixed methods. In some cases, the second variable is introduced in the formulation of the problem because of its physical interest and it is usually related with some derivatives of the original variable. This is the case, for example, in the elasticity equations, where the stress can be introduced to be approximated at the same time as the displacement. In other cases there are two natural independent variables and so, the mixed formulation is the natural one. This is the case of the Stokes equations, where the two variables are the velocity and the pressure.

The mathematical analysis and applications of mixed finite element methods have been widely developed since the seventies. A general analysis for this kind of methods was first developed by Brezzi [13]. We also have to mention the papers by Babuska [9] and by Crouzeix and Raviart [22] which, although for particular problems, introduced some of the fundamental ideas for the analysis of mixed methods. We also refer the reader to [32, 31], where general results were obtained, and to the books [17, 45, 37].

The rest of this work is organized as follows: in Section 2 we review some basic tools for the analysis of finite element methods. Section 3 deals with the mixed formulation of second order elliptic problems and their finite element approximation. We introduce the Raviart-Thomas spaces [44, 49, 41] and their generalization to higher dimensions, prove some of their basic properties, and construct the Raviart-Thomas interpolation operator which is a basic tool for the analysis of mixed methods. Then, we prove optimal order error estimates and a superconvergence result for the scalar variable.

*Departamento de Matemática, Facultad de Ciencias Exactas, Universidad de Buenos Aires, 1428 Buenos Aires, Argentina. E-mail: rduran@dm.uba.ar 
We follow the ideas developed in several papers (see for example $[24,16]$ ). Although for simplicity we consider the Raviart-Thomas spaces, the error analysis depends only on some basic properties of the spaces and the interpolation operator, and therefore, analogous results hold for approximations obtained with other finite element spaces. We end the section recalling other known families of spaces and giving some references. In Section 4 we introduce an a posteriori error estimator and prove its equivalence with an appropriate norm of the error up to higher order terms. For simplicity, we present the a posteriori error analysis only in the two dimensional case. Finally, in Section 5, we introduce the general abstract setting for mixed formulations and prove general existence and approximation results.

\section{Preliminary results}

In this section we recall some basic results for the analysis of finite element approximations.

We will use the standard notation for Sobolev spaces and their norms, namely, given a domain $\Omega \subset \mathbb{R}^{n}$ and any positive integer $k$

$$
H^{k}(\Omega)=\left\{\phi \in L^{2}(\Omega): D^{\alpha} \phi \in L^{2}(\Omega) \quad \forall|\alpha| \leq k\right\},
$$

where

$$
\alpha=\left(\alpha_{1}, \cdots, \alpha_{n}\right) \quad, \quad|\alpha|=\alpha_{1}+\cdots+\alpha_{n} \quad \text { and } \quad D^{\alpha} \phi=\frac{\partial^{|\alpha|} \phi}{\partial x_{1}^{\alpha_{1}} \cdots \partial x_{n}^{\alpha_{n}}}
$$

and the derivatives are taken in the distributional or weak sense.

$H^{k}(\Omega)$ is a Hilbert space with the norm given by

$$
\|\phi\|_{H^{k}(\Omega)}^{2}=\sum_{|\alpha| \leq k}\left\|D^{\alpha} \phi\right\|_{L^{2}(\Omega)}^{2} .
$$

Given $\phi \in H^{k}(\Omega)$ and $j \in \mathbb{N}$ such $1 \leq j \leq k$ we define $\nabla^{j} \phi$ by

$$
\left|\nabla^{j} \phi\right|^{2}=\sum_{|\alpha|=j}\left|D^{\alpha} \phi\right|^{2}
$$

Analogous notations will be used for vector fields, i.e., if $\mathbf{v}=\left(v_{1}, \cdots, v_{n}\right)$ then $D^{\alpha} \mathbf{v}=\left(D^{\alpha} v_{1}, \cdots, D^{\alpha} v_{n}\right)$ and

$$
\|\mathbf{v}\|_{H^{k}(\Omega)}^{2}=\sum_{i=1}^{n}\left\|v_{i}\right\|_{H^{k}(\Omega)}^{2} \quad \text { and } \quad\left|\nabla^{j} \mathbf{v}\right|^{2}=\sum_{i=1}^{n}\left|\nabla^{j} v_{i}\right|^{2}
$$


We will also work with the following subspaces of $H^{1}(\Omega)$ :

$$
\begin{gathered}
H_{0}^{1}(\Omega)=\left\{\phi \in H^{1}(\Omega):\left.\phi\right|_{\partial \Omega}=0\right\}, \\
\widehat{H}^{1}(\Omega)=\left\{\phi \in H^{1}(\Omega): \int_{\Omega} \phi d x=0\right\} .
\end{gathered}
$$

Also, we will use the standard notation $\mathcal{P}_{k}$ for the space of polynomials of degree less than or equal to $k$ and, if $x \in \mathbb{R}^{n}$ and $\alpha$ is a multi-index, we will set $x^{\alpha}=x_{1}^{\alpha_{1}} \cdots x_{n}^{\alpha_{n}}$.

The letter $C$ will denote a generic constant not necessarily the same at each occurrence.

Given a function in a Sobolev space of a domain $\Omega$ it is important to know whether it can be restricted to $\partial \Omega$, and conversely, when can a function defined on $\partial \Omega$ be extended to $\Omega$ in such a way that it belongs to the original Sobolev space. We will use the following trace theorem. We refer the reader for example to $[38,33]$ for the proof of this theorem and for the definition of the fractional-order Sobolev space $H^{\frac{1}{2}}(\partial \Omega)$.

Theorem 2.1 Given $\phi \in H^{1}(\Omega)$, where $\Omega \subset \mathbb{R}^{n}$ is a Lipschitz domain, there exists a constant $C$ depending only on $\Omega$ such that

$$
\|\phi\|_{H^{\frac{1}{2}(\partial \Omega)}} \leq C\|\phi\|_{H^{1}(\Omega)} .
$$

In particular,

$$
\|\phi\|_{L^{2}(\partial \Omega)} \leq C\|\phi\|_{H^{1}(\Omega)} .
$$

Moreover, if $g \in H^{\frac{1}{2}}(\partial \Omega)$, there exists $\phi \in H^{1}(\Omega)$ such that $\left.\phi\right|_{\partial \Omega}=g$ and

$$
\|\phi\|_{H^{1}(\Omega)} \leq C\|g\|_{H^{\frac{1}{2}(\partial \Omega)}} .
$$

One of the most important results in the analysis of variational methods for elliptic problems is the Friedrichs-Poincaré inequality for functions with vanishing mean average, that we state below (see for example [36] for the case of Lipschitz domains and [43] for another proof in the case of convex domains). Assume that $\Omega$ is a Lipschitz domain. Then, there exists a constant $C$ depending only on the domain $\Omega$ such that for any $f \in \widehat{H}^{1}(\Omega)$,

$$
\|f\|_{L^{2}(\Omega)} \leq C\|\nabla f\|_{L^{2}(\Omega)} .
$$

The Friedrichs-Poincaré inequality can be seen as a particular case of the next result on polynomial approximation which is basic in the analysis of finite element methods. 
Several different arguments have been given for the proof of the next lemma. See for example $[12,25,26,51]$. Here we give a nice argument which, to our knowledge, is due to M. Dobrowolski for the lowest order case on convex domains (and as far as we know has not been published). The proof given here for the case of domains which are star-shaped with respect to a subset of positive measure and any degree of approximation is an immediate extension of Dobrowolski's argument. For simplicity we present the proof for the $L^{2}$-case (which is the case that we will use), but the reader can check that an analogous argument applies for $L^{p}$ based Sobolev spaces $(1 \leq p<\infty)$.

Assume that $\Omega$ is star-shaped with respect to a set $B \subseteq \Omega$ of positive measure. Given an integer $k \geq 0$ and $f \in H^{k+1}(\Omega)$ we introduce the averaged Taylor polynomial approximation of $f, Q_{k, B} f \in \mathcal{P}_{k}$ defined by

$$
Q_{k} f(x)=\frac{1}{|B|} \int_{B} T_{k} f(y, x) d y
$$

where $T_{k} f(y, x)$ is the Taylor expansion of $f$ centered at $y$, namely,

$$
T_{k} f(y, x)=\sum_{|\alpha| \leq k} D^{\alpha} f(y) \frac{(x-y)^{\alpha}}{\alpha !} .
$$

Lemma 2.2 Let $\Omega \subset \mathbb{R}^{n}$ be a domain with diameter $d$ which is star-shaped with respect to a set of positive measure $B \subset \Omega$. Given an integer $k \geq 0$ and $f \in H^{k+1}(\Omega)$, there exists a constant $C=C(k, n)$ such that, for $0 \leq|\beta| \leq$ $k+1$

$$
\left\|D^{\beta}\left(f-Q_{k, B} f\right)\right\|_{L^{2}(\Omega)} \leq C \frac{|\Omega|^{1 / 2}}{|B|^{1 / 2}} d^{k+1-|\beta|}\left\|\nabla^{k+1} f\right\|_{L^{2}(\Omega)} .
$$

In particular, if $\Omega$ is convex,

$$
\left\|D^{\beta}\left(f-Q_{k, \Omega} f\right)\right\|_{L^{2}(\Omega)} \leq C d^{k+1-|\beta|}\left\|\nabla^{k+1} f\right\|_{L^{2}(\Omega)} .
$$

Proof. By density we can assume that $f \in C^{\infty}(\Omega)$. Then we can write

$$
f(x)-T_{k} f(y, x)=(k+1) \sum_{|\alpha|=k+1} \frac{(x-y)^{\alpha}}{\alpha !} \int_{0}^{1} D^{\alpha} f(t y+(1-t) x) t^{k} d t .
$$


Integrating this inequality over $B$ (in the variable $y$ ) and dividing by $|B|$ we have

$f(x)-Q_{k, B} f(x)=\frac{k+1}{|B|} \sum_{|\alpha|=k+1} \int_{B} \int_{0}^{1} \frac{(x-y)^{\alpha}}{\alpha !} D^{\alpha} f(t y+(1-t) x) t^{k} d t d y$

and so,

$\int_{\Omega}\left|f(x)-Q_{k, B} f(x)\right|^{2} d x \leq C \frac{d^{2(k+1)}}{|B|^{2}} \sum_{|\alpha|=k+1} \int_{\Omega}\left(\int_{B} \int_{0}^{1}\left|D^{\alpha} f(t y+(1-t) x)\right| t^{k} d t d y\right)^{2} d x$

$\leq C \frac{d^{2(k+1)}}{|B|^{2}} \sum_{|\alpha|=k+1} \int_{\Omega}\left(\int_{B} \int_{0}^{1}\left|D^{\alpha} f(t y+(1-t) x)\right|^{2} d t d y\right)\left(\int_{B} \int_{0}^{1} t^{2 k} d t d y\right) d x$.

Therefore,

$\int_{\Omega}\left|f(x)-Q_{k, B} f(x)\right|^{2} d x \leq C \frac{d^{2(k+1)}}{|B|} \sum_{|\alpha|=k+1} \int_{\Omega} \int_{B} \int_{0}^{1}\left|D^{\alpha} f(t y+(1-t) x)\right|^{2} d t d y d x$

Now, for each $\alpha$,

$$
\begin{gathered}
\int_{\Omega} \int_{B} \int_{0}^{1}\left|D^{\alpha} f(t y+(1-t) x)\right|^{2} d t d y d x \\
=\int_{\Omega} \int_{B} \int_{0}^{\frac{1}{2}}\left|D^{\alpha} f(t y+(1-t) x)\right|^{2} d t d y d x+\int_{\Omega} \int_{B} \int_{\frac{1}{2}}^{1}\left|D^{\alpha} f(t y+(1-t) x)\right|^{2} d t d y d x=: I+I I
\end{gathered}
$$

Let us call $g_{\alpha}$ the extension by zero of $D^{\alpha} f$ to $\mathbb{R}^{n}$. Then, by Fubini's theorem and two changes of variables we have

$$
\begin{aligned}
I \leq & \int_{B} \int_{0}^{\frac{1}{2}} \int_{\mathbb{R}^{n}}\left|g_{\alpha}(t y+(1-t) x)\right|^{2} d x d t d y=\int_{B} \int_{0}^{\frac{1}{2}} \int_{\mathbb{R}^{n}}\left|g_{\alpha}((1-t) x)\right|^{2} d x d t d y \\
& =\int_{B} \int_{0}^{\frac{1}{2}} \int_{\mathbb{R}^{n}}\left|g_{\alpha}(z)\right|^{2}(1-t)^{-n} d z d t d y \leq 2^{n-1}|B| \int_{\Omega}\left|D^{\alpha} f(z)\right|^{2} d z .
\end{aligned}
$$

Analogously,

$$
\begin{gathered}
I I \leq \int_{\Omega} \int_{\frac{1}{2}}^{1} \int_{\mathbb{R}^{n}}\left|g_{\alpha}(t y+(1-t) x)\right|^{2} d y d t d x=\int_{\Omega} \int_{\frac{1}{2}}^{1} \int_{\mathbb{R}^{n}}\left|g_{\alpha}(t y)\right|^{2} d y d t d x \\
=\int_{\Omega} \int_{\frac{1}{2}}^{1} \int_{\mathbb{R}^{n}}\left|g_{\alpha}(z)\right|^{2} t^{-n} d z d t d x \leq 2^{n-1}|\Omega| \int_{\Omega}\left|D^{\alpha} f(z)\right|^{2} d z .
\end{gathered}
$$


Therefore, replacing these bounds in (2.5) we obtain (2.3) for $\beta=0$.

On the other hand, an elementary computation shows that

$$
D^{\beta} Q_{k, B} f(x)=Q_{k-|\beta|, B}\left(D^{\beta} f\right)(x) \quad \forall|\beta| \leq k
$$

and therefore, the estimate (2.3) for $|\beta|>0$ follows from the case $\beta=0$ applied to $D^{\beta} f$.

An important consequence of this result are the following error estimates for the $L^{2}$-projection onto $\mathcal{P}_{m}$.

Corollary 2.3 Let $\Omega \subset \mathbb{R}^{n}$ be a domain with diameter $d$ which is starshaped with respect to a set of positive measure $B \subset \Omega$. Given an integer $m \geq 0$, let $P: L^{2}(\Omega) \rightarrow \mathcal{P}_{m}$ be the $L^{2}$-orthogonal projection. There exists a constant $C=C(j, n)$ such that, for $0 \leq j \leq m$, if $f \in H^{j}(\Omega)$, then

$$
\|f-P f\|_{L^{2}(\Omega)} \leq C \frac{|\Omega|^{1 / 2}}{|B|^{1 / 2}} d^{j}\left|\nabla^{j} f\right|_{L^{2}(\Omega)} .
$$

Remark 2.1 Analogous results to Lemma 2.3 and its corollary hold for bounded Lipschitz domains because this kind of domains can be written as a finite union of star-shaped domains (see [25] for details).

The following result is fundamental in the analysis of mixed finite element approximations.

Lemma 2.4 Let $\Omega \subset \mathbb{R}^{n}$ be a bounded domain. Given $f \in L^{2}(\Omega)$ there exists $\mathbf{v} \in H^{1}(\Omega)^{n}$ such that

$$
\operatorname{div} \mathbf{v}=f \quad \text { in } \Omega
$$

and

$$
\|\mathbf{v}\|_{H^{1}(\Omega)} \leq C\|f\|_{L^{2}(\Omega)}
$$

with a constant $C$ depending only on $\Omega$.

Proof. Let $B \in \mathbb{R}^{n}$ be a ball containing $\Omega$ and $\phi$ be the solution of the boundary problem

$$
\left\{\begin{aligned}
\Delta \phi=f & \text { in } B \\
\phi=0 & \text { on } \partial B
\end{aligned}\right.
$$

It is known that $\phi$ satisfies the following a priori estimate (see for example [36])

$$
\|\phi\|_{H^{2}(\Omega)} \leq C\|f\|_{L^{2}(\Omega)}
$$

and therefore $\mathbf{v}=\nabla \phi$ satisfies (2.6) and (2.7). 
Remark 2.2 To treat Neumann boundary conditions we would need the existence of a solution of $\operatorname{div} \mathbf{v}=f$ satisfying (2.7) and the boundary condition $\mathbf{v} \cdot \mathbf{n}=0$ on $\partial \Omega$. Such $a \mathbf{v}$ can be obtained by solving a Neumann problem in $\Omega$ for smooth domains or convex polygonal or polyhedral domains. For more general domains, including arbitrary polygonal or polyhedral domains, the existence of $\mathbf{v}$ satisfying (2.6) and (2.7) can be proved in different ways. In fact $\mathbf{v}$ can be taken such that all its components vanish on $\partial \Omega$ (see for example [2, 7, 30]).

A usual technique to obtain error estimates for finite element approximations is to work in a reference element and then change variables to prove results for a general element. Let us introduce some notations and recall some basic estimates.

Fix a reference simplex $\widehat{T} \subset \mathbb{R}^{n}$. Given a simplex $T \subset \mathbb{R}^{n}$, there exists an invertible affine map $F: \widehat{T} \rightarrow T, F(\hat{x})=A \hat{x}+b$, with $A \in \mathbb{R}^{n \times n}$ and $b \in \mathbb{R}^{n}$.

We call $h_{T}$ the diameter of $T$ and $\rho_{T}$ the diameter of the largest ball inscribed in $T$ (see Figure 1). We will use the regularity assumption on the elements, namely, many of our estimates will depend on a constant $\sigma$ such that

$$
\frac{h_{T}}{\rho_{T}} \leq \sigma
$$
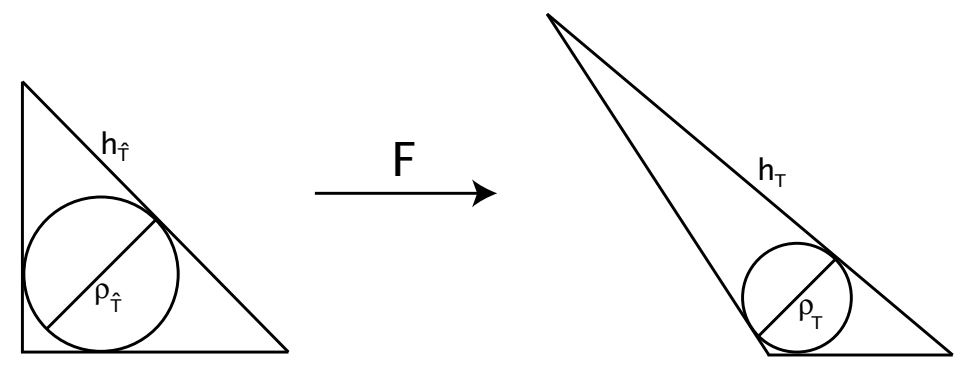

Figure 1

It is known that (see [19]), for the matrix norm associated with the euclidean vector norm, the following estimates hold:

$$
\|A\| \leq \frac{h_{T}}{\rho_{\widehat{T}}} \quad \text { and } \quad\left\|A^{-1}\right\| \leq \frac{h_{\widehat{T}}}{\rho_{T}}
$$

With any $\phi \in L^{2}(T)$ we associate $\hat{\phi} \in L^{2}(\widehat{T})$ in the usual way, namely,

$$
\phi(x)=\hat{\phi}(\hat{x})
$$


where $x=F(\hat{x})$.

We end this section by recalling the so-called inverse estimates which are a fundamental tool in finite element analysis. We give only a particular case which will be needed for our proofs (see for example [19] for more general inverse estimates).

Lemma 2.5 Given a simplex $T$ there exists a constant $C=C(\sigma, k, n, \widehat{T})$ such that, for any $p \in \mathcal{P}_{k}(T)$,

$$
\|\nabla p\|_{L^{2}(T)} \leq \frac{C}{h_{T}}\|p\|_{L^{2}(T)} .
$$

Proof. Since $\mathcal{P}_{k}(\widehat{T})$ is a finite dimensional space, all the norms defined on it are equivalent. In particular, there exists a constant $\widehat{C}$ depending on $k$ and $\widehat{T}$ such that

$$
\|\widehat{\nabla} \hat{p}\|_{L^{2}(\widehat{T})} \leq \widehat{C}\|\hat{p}\|_{L^{2}(\widehat{T})}
$$

for any $\hat{p} \in \mathcal{P}_{k}(\widehat{T})$.

An easy computation shows that

$$
\nabla p=A^{-T} \hat{\nabla} \hat{p}
$$

where $A^{-T}$ is the transpose matrix of $A^{-1}$. Therefore, using the bound for $\left\|A^{-1}\right\|$ given in (2.10) together with (2.12) and (2.9) we have

$$
\begin{aligned}
& \int_{T}|\nabla p|^{2} d x=\int_{\widehat{T}}\left|A^{-T} \widehat{\nabla} \hat{p}\right|^{2}|\operatorname{det} A| d \hat{x} \leq\left\|A^{-1}\right\|^{2} \int_{\widehat{T}}|\widehat{\nabla} \hat{p}|^{2}|\operatorname{det} A| d \hat{x} \\
\leq & \widehat{C} \frac{h_{\widehat{T}}^{2}}{\rho_{T}^{2}} \int_{\widehat{T}}|\hat{p}|^{2}|\operatorname{det} A| d \hat{x}=\widehat{C} \frac{h_{\widehat{T}}^{2}}{\rho_{T}^{2}} \int_{T}|p|^{2} d x \leq \widehat{C} \sigma^{2} \frac{h_{\widehat{T}}^{2}}{h_{T}^{2}} \int_{T}|p|^{2} d x .
\end{aligned}
$$

\section{Mixed approximation of second order elliptic problems}

In this section we introduce the mixed finite element approximation of second order elliptic problems and we develop the a priori error analysis. We consider the so called $h$-version of the finite element method, namely, fixing a degree of approximation we prove error estimates in terms of the mesh 
size. We present the error analysis for the case of $L^{2}$ based norms (following essentially [24]) and refer to [27, 34, 35] for error estimates in other norms.

As it is usually done, we prove error estimates for any degree of approximation under the hypothesis that the solution is regular enough in order to show the best possible order of a method. However, the reader has to be aware that, in practice, for polygonal or polyhedral domains (which is the case considered here!) the solution is in general not smooth due to singularities at the angles and therefore the order of convergence is limited by the regularity of the solution of each particular problem considered. On the other hand, for domains with smooth boundary where the solutions might be very regular, a further error analysis considering the approximation of the boundary is needed.

Consider the elliptic problem

$$
\left\{\begin{aligned}
-\operatorname{div}(a \nabla p) & =f & & \text { in } \Omega \\
p & =0 & & \text { on } \partial \Omega
\end{aligned}\right.
$$

where $\Omega \subset \mathbb{R}^{n}$ is a polyhedral domain and $a=a(x)$ is a function bounded by above and below by positive constants.

In many applications the variable of interest is

$$
\mathbf{u}=-a \nabla p
$$

and then, it could be desirable to use a mixed finite element method which approximates $\mathbf{u}$ and $p$ simultaneously. With this purpose, problem (3.1) is decomposed into a first order system as follows:

$$
\left\{\begin{array}{rlrl}
\mathbf{u}+a \nabla p & =0 & & \text { in } \Omega \\
\operatorname{div} \mathbf{u}=f & & \text { in } \Omega \\
p=0 & & \text { on } \partial \Omega
\end{array}\right.
$$

To write an appropriate weak formulation of this problem we introduce the space

$$
H(\operatorname{div}, \Omega)=\left\{\mathbf{v} \in L^{2}(\Omega)^{n}: \operatorname{div} \mathbf{v} \in L^{2}(\Omega)\right\}
$$

which is a Hilbert space with norm given by

$$
\|\mathbf{v}\|_{H(\operatorname{div}, \Omega)}^{2}=\|\mathbf{v}\|_{L^{2}(\Omega)}^{2}+\|\operatorname{div} \mathbf{v}\|_{L^{2}(\Omega)}^{2} .
$$

Defining $\mu(x)=1 / a(x)$, the first equation in (3.2) can be rewritten as

$$
\mu \mathbf{u}+\nabla p=0 \text { in } \Omega .
$$


Multiplying by test functions and integrating by parts we obtain the standard weak mixed formulation of problem (3.2), namely,

$$
\left\{\begin{aligned}
\int_{\Omega} \mu \mathbf{u} \cdot \mathbf{v} d x-\int_{\Omega} p \operatorname{div} \mathbf{v} d x=0 & \forall \mathbf{v} \in H(\operatorname{div}, \Omega) \\
\int_{\Omega} q \operatorname{div} \mathbf{u} d x=\int_{\Omega} f q d x & \forall q \in L^{2}(\Omega)
\end{aligned}\right.
$$

Observe that the Dirichlet boundary condition is implicit in the weak formulation (i.e., it is the type of condition usually called natural). Instead, Neumann boundary conditions would have to be imposed on the space (essential conditions). This is exactly opposite to what happens in the case of standard formulations.

The weak formulation (3.3) involves the divergence of the solution and of the test functions but not arbitrary first derivatives. This fact allows us to work on the space $H(\operatorname{div}, \Omega)$ instead of the smaller $H^{1}(\Omega)^{n}$ and this will be important for the finite element approximation because piecewise polynomials vector functions do not need to have both components continuous to be in $H(\operatorname{div}, \Omega)$, but only their normal component.

In order to define finite element approximations to the solution $(\mathbf{u}, p)$ of (3.3) we need to introduce finite dimensional subspaces of $H(\operatorname{div}, \Omega)$ and $L^{2}(\Omega)$ made of piecewise polynomial functions.

For simplicity we will consider the case of triangular elements (or its generalizations to higher dimensions) and the associated Raviart-Thomas spaces which are the best-known spaces for this problem. This family of spaces was introduced in [44] in the two dimensional case, while its extension to three dimensions was first considered in [41]. Since no essential technical difficulties arise in the general case, we prefer to present the spaces and the analysis of their properties in the general n-dimensional case (although, of course, we are mainly interested in the cases $n=2$ and $n=3$ ). Below we will comment and give references on different variants of spaces.

First we introduce the local spaces, analyze their properties and construct the Raviart-Thomas interpolation.

Given a simplex $T \in \mathbb{R}^{n}$, the local Raviart-Thomas space $[44,41]$ of order $k \geq 0$ is defined by

$$
\mathcal{R} \mathcal{T}_{k}(T)=\mathcal{P}_{k}(T)^{n}+x \mathcal{P}_{k}(T)
$$

In the following lemma we give some basic properties of the spaces $\mathcal{R} \mathcal{T}_{k}(T)$. We denote with $F_{i}, i=1, \cdots, n+1$, the faces of a simplex $T$ and with $\mathbf{n}_{i}$ their corresponding exterior normals. 
Lemma $3.1 \quad$ a) $\operatorname{dim} \mathcal{R} \mathcal{T}_{k}(T)=n\left(\begin{array}{c}k+n \\ k\end{array}\right)+\left(\begin{array}{c}k+n-1 \\ k\end{array}\right)$

b) If $\mathbf{v} \in \mathcal{R T}_{k}(T)$ then, $\mathbf{v} \cdot \mathbf{n}_{i} \in \mathcal{P}_{k}\left(F_{i}\right)$ for $i=1, \cdots, n+1$

c) If $\mathbf{v} \in \mathcal{R T}_{k}(T)$ is such that $\operatorname{div} \mathbf{v}=0$ then, $\mathbf{v} \in \mathcal{P}_{k}^{n}$

Proof. Any $\mathbf{v} \in \mathcal{R} \mathcal{T}_{k}(T)$ can be written as

$$
\mathbf{v}=\mathbf{w}+x \sum_{|\alpha|=k} a_{\alpha} x^{\alpha}
$$

with $\mathbf{w} \in \mathcal{P}_{k}^{n}$.

Recall that $\operatorname{dim} \mathcal{P}_{k}=\left(\begin{array}{c}k+n \\ k\end{array}\right)$ and that the number of multi-indeces $\alpha$ such that $|\alpha|=k$ is $\left(\begin{array}{c}k+n-1 \\ k\end{array}\right)$. Then, $\left.a\right)$ follows from (3.5).

Now, the face $F_{i}$ is on a hyperplane of equation $x \cdot \mathbf{n}_{i}=s$ with $s \in \mathbb{R}$. Therefore, if $\mathbf{v}=\mathbf{w}+x p$ with $\mathbf{w} \in \mathcal{P}_{k}^{n}$ and $p \in \mathcal{P}_{k}$, we have

$$
\mathbf{v} \cdot \mathbf{n}_{i}=\mathbf{w} \cdot \mathbf{n}_{i}+x \cdot \mathbf{n}_{i} p=\mathbf{w} \cdot \mathbf{n}_{i}+s p \in \mathcal{P}_{k}
$$

which proves $b$ ).

Finally, if $\operatorname{div} \mathbf{v}=0$ we take the divergence in the expression (3.5) and conclude easily that $a_{\alpha}=0$ for all $\alpha$ and therefore $c$ ) holds.

Our next goal is to construct an interpolation operator

$$
\Pi_{T}: H^{1}(T)^{n} \rightarrow \mathcal{R} \mathcal{T}_{k}
$$

which will be fundamental for the error analysis. We fix $k$ and to simplify notation we omit the index $k$ in the operator.

For simplicity we define the interpolation for functions in $H^{1}(T)^{n}$ although it is possible (and necessary in many cases!) to do the same construction for less regular functions. Indeed, the reader who is familiar with fractional order Sobolev spaces and trace theorems will realize that the degrees of freedom defining the interpolation are well defined for functions in $H^{s}(T)^{n}$, with $s>1 / 2$.

The local interpolation operator is defined in the following lemma.

Lemma 3.2 Given $\mathbf{v} \in H^{1}(T)^{n}$, where $T \in \mathbb{R}^{n}$ is a simplex, there exists a unique $\Pi_{T} \mathbf{v} \in \mathcal{R} \mathcal{T}_{k}(T)$ such that

$$
\int_{F_{i}} \Pi_{T} \mathbf{v} \cdot \mathbf{n}_{i} p_{k} d s=\int_{F_{i}} \mathbf{v} \cdot \mathbf{n}_{i} p_{k} d s \quad \forall p_{k} \in \mathcal{P}_{k}\left(F_{i}\right), \quad i=1, \cdots, n+1
$$


and, if $k \geq 1$,

$$
\int_{T} \Pi_{T} \mathbf{v} \cdot \mathbf{p}_{k-1} d x=\int_{T} \mathbf{v} \cdot \mathbf{p}_{k-1} d x \quad \forall \mathbf{p}_{k-1} \in \mathcal{P}_{k-1}^{n}(T)
$$

Proof. First, we want to see that the number of conditions defining $\Pi_{T} \mathbf{v}$ equals the dimension of $\mathcal{R} \mathcal{T}_{k}(T)$. This is easily verified for the case $k=0$, so let us consider the case $k \geq 1$.

Since $\operatorname{dim} \mathcal{P}_{k}\left(F_{i}\right)=\left(\begin{array}{c}k+n-1 \\ k\end{array}\right)$, the number of conditions in $(3.6)$ is

$$
\# \text { of faces } \times \operatorname{dim} \mathcal{P}_{k}\left(F_{i}\right)=(n+1)\left(\begin{array}{c}
k+n-1 \\
k
\end{array}\right) .
$$

On the other hand, the number of conditions in (3.7) is

$$
\operatorname{dim} \mathcal{P}_{k-1}^{n}(T)=n\left(\begin{array}{c}
k+n-1 \\
k-1
\end{array}\right)
$$

Then, the total number of conditions defining $\Pi_{T} \mathbf{v}$ is

$$
(n+1)\left(\begin{array}{c}
k+n-1 \\
k
\end{array}\right)+n\left(\begin{array}{c}
k+n-1 \\
k-1
\end{array}\right) .
$$

Therefore, in view of $a$ ) of Lemma (3.1), we have to check that

$$
n\left(\begin{array}{c}
k+n \\
k
\end{array}\right)+\left(\begin{array}{c}
k+n-1 \\
k
\end{array}\right)=(n+1)\left(\begin{array}{c}
k+n-1 \\
k
\end{array}\right)+n\left(\begin{array}{c}
k+n-1 \\
k-1
\end{array}\right)
$$

or equivalently,

$$
\left(\begin{array}{c}
k+n \\
k
\end{array}\right)=\left(\begin{array}{c}
k+n-1 \\
k
\end{array}\right)+\left(\begin{array}{c}
k+n-1 \\
k-1
\end{array}\right)
$$

which can be easily verified.

Therefore, in order to show the existence of $\Pi_{T} \mathbf{v}$, it is enough to prove uniqueness. So, take $\mathbf{v} \in \mathcal{R} \mathcal{T}_{k}(T)$ such that

$$
\int_{F_{i}} \mathbf{v} \cdot \mathbf{n}_{i} p_{k} d s=0 \quad \forall p_{k} \in \mathcal{P}_{k}\left(F_{i}\right), \quad i=1, \cdots, n+1
$$


and

$$
\int_{T} \mathbf{v} \cdot \mathbf{p}_{k-1} d x=0 \quad \forall \mathbf{p}_{k-1} \in \mathcal{P}_{k-1}^{n}(T)
$$

From $b$ ) of Lemma 3.1 and (3.8) it follows that $\mathbf{v} \cdot \mathbf{n}_{i}=0$ on $F_{i}$. Then, using now (3.9) we have

$$
\int_{T}(\operatorname{div} \mathbf{v})^{2} d x=-\int_{T} \mathbf{v} \cdot \nabla(\operatorname{div} \mathbf{v}) d x=0
$$

because $\nabla(\operatorname{div} \mathbf{v}) \in \mathcal{P}_{k-1}^{n}(T)$. Consequently $\operatorname{div} \mathbf{v}=0$ and so, from $\left.c\right)$ of Lemma 3.1 we know that $\mathbf{v} \in \mathcal{P}_{k}^{n}(T)$.

Therefore, for each $i=1, \cdots, n+1$, the component $\mathbf{v} \cdot \mathbf{n}_{i}$ is a polynomial of degree $k$ on $T$ which vanishes on $F_{i}$. Therefore, calling $\lambda_{i}$ the barycentric coordinates associated with $T$ (i.e., $\lambda_{i}(x)=0$ on $F_{i}$ ), we have

$$
\mathbf{v} \cdot \mathbf{n}_{i}=\lambda_{i} q_{k-1}
$$

with $q_{k-1} \in \mathcal{P}_{k-1}(T)$. But, from (3.9) we know that

$$
\int_{T} \mathbf{v} \cdot \mathbf{n}_{i} p_{k-1} d x=0 \quad \forall p_{k-1} \in \mathcal{P}_{k-1}(T)
$$

and choosing $p_{k-1}=q_{k-1}$ we obtain

$$
\int_{T} \lambda_{i} q_{k-1}^{2} d x=0
$$

Therefore, since $\lambda_{i}$ does not change sign on $T$, it follows that $q_{k-1}=0$ and consequently $\mathbf{v} \cdot \mathbf{n}_{i}=0$ in $T$ for $i=1, \cdots, n+1$. In particular, there are $n$ linearly independent directions in which $\mathbf{v}$ has vanishing components and, therefore, $\mathbf{v}=0$ as we wanted to see.

Figure 2 shows the degrees of freedom defining $\Pi_{T}$ for $k=0$ and $k=1$ in the two dimensional case. The arrows indicate normal components values and the filled circle, values of $\mathbf{v}$ (and so it corresponds to two degrees of freedom).

To obtain error estimates for the mixed finite element approximations we need to know the approximation properties of the Raviart-Thomas interpolation $\Pi_{T}$. The analysis given in $[44,49]$ makes use of general standard arguments for polynomial-preserving operators (see [19]). The main difference with the error analysis for Lagrange interpolation is that here we have to use an appropriate transformation, known as the Piola transform, which preserves the degrees of freedom defining $\Pi_{T} \mathbf{v}$. 

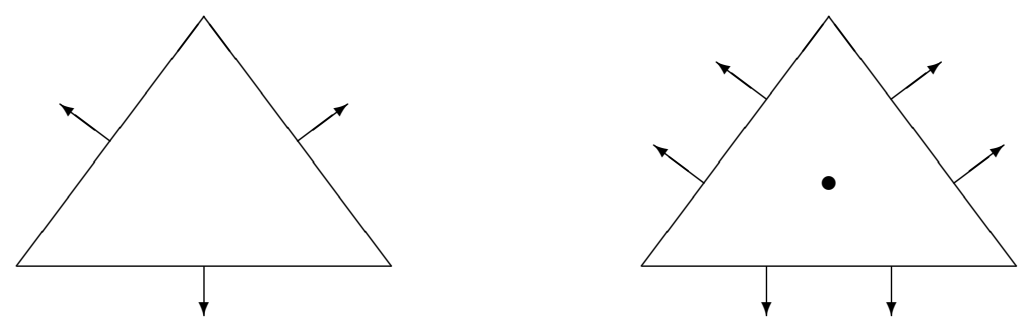

Figure 2: Degrees of freedom for $\mathcal{R} \mathcal{T}_{0}$ and $\mathcal{R} \mathcal{T}_{1}$ in $\mathbb{R}^{2}$

The Piola transform is defined in the following way. Given two domains $\widehat{\Omega}, \Omega \subset \mathbb{R}^{n}$ and a bijective map $F: \widehat{\Omega} \rightarrow \Omega$, let $D F$ be the Jacobian matrix of $F$ and $J:=\operatorname{det} D F$. Assume that $J$ does not vanish at any point, then, we define for $\hat{\mathbf{v}} \in L^{2}(\widehat{\Omega})^{n}$

$$
\mathbf{v}(x)=\frac{1}{|J(\hat{x})|} \widehat{D} F(\hat{x}) \hat{\mathbf{v}}(\hat{x})
$$

where $x=F(\hat{x})$. Here and in what follows, the hat over differential operators indicates that the derivatives are taken with respect to $\hat{x}$.

We recall that scalar functions are transformed as indicated in (2.11) (we are using the same notation for the transformation of vector and scalar functions since no confusion is possible).

In the particular case that $F$ is an affine map given by $A \hat{x}+b$ we have $J=\operatorname{det} A$ and

$$
\mathbf{v}(x)=\frac{1}{|J|} A \hat{\mathbf{v}}(\hat{x}) .
$$

In the next lemma we give some fundamental properties of the Piola transform. For simplicity, we prove the results only for affine transformations, which is the useful case for our purposes. However, it is important to remark that analogous results hold for general transformations and this is important, for example, to work with general quadrilateral elements.

Lemma 3.3 If $\mathbf{v} \in H(\operatorname{div}, T)$ and $\phi \in H^{1}(T)$ then

$$
\int_{T} \operatorname{div} \mathbf{v} \phi d x=\int_{\widehat{T}} \widehat{\operatorname{div}} \hat{\mathbf{v}} \hat{\phi} d \hat{x},
$$




$$
\int_{T} \mathbf{v} \cdot \nabla \phi d x=\int_{\widehat{T}} \hat{\mathbf{v}} \cdot \hat{\nabla} \hat{\phi} d \hat{x}
$$

and

$$
\int_{\partial T} \mathbf{v} \cdot \mathbf{n} \phi d s=\int_{\partial \widehat{T}} \hat{\mathbf{v}} \cdot \hat{\mathbf{n}} \hat{\phi} d \hat{s} .
$$

Proof. From the definition of the Piola transform (3.10) we have

$$
D \mathbf{v}(x)=\frac{1}{|J|} A D\left(\hat{\mathbf{v}} \circ F^{-1}\right)(x)=\frac{1}{|J|} A \widehat{D} \hat{\mathbf{v}}(\hat{x}) D F^{-1}(x)=\frac{1}{|J|} A \widehat{D} \hat{\mathbf{v}}(\hat{x}) A^{-1} .
$$

Then,

$$
\operatorname{div} \mathbf{v}=\operatorname{tr} D \mathbf{v}=\frac{1}{|J|} \operatorname{tr}\left(A \widehat{D} \hat{\mathbf{v}} A^{-1}\right)=\frac{1}{|J|} \operatorname{tr} \widehat{D} \hat{\mathbf{v}}=\frac{1}{|J|} \widehat{\operatorname{div}} \hat{\mathbf{v}}
$$

and therefore (3.11) follows by a change of variable.

To prove (3.12) recall that

$$
\nabla \phi=A^{-T} \hat{\nabla} \hat{\phi}
$$

Then,

$$
\int_{T} \mathbf{v} \cdot \nabla \phi d x=\int_{\widehat{T}} A \hat{\mathbf{v}} \cdot A^{-T} \hat{\nabla} \hat{\phi} d \hat{x}=\int_{\widehat{T}} \hat{\mathbf{v}} \cdot \hat{\nabla} \hat{\phi} d \hat{x} .
$$

Finally,(3.13) follows from (3.11) and (3.12) applying the divergence theorem.

Remark 3.1 The integral over $\partial T$ in the previous lemma has to be understood as a duality product between $\mathbf{v} \cdot \mathbf{n} \in H^{-\frac{1}{2}}(\partial T)$ and $\phi \in H^{\frac{1}{2}}(\partial T)$.

We can now prove the invariance of the Raviart-Thomas interpolation under the Piola transform.

Lemma 3.4 Given a simplex $T \in \mathbb{R}^{n}$ and $\mathbf{v} \in H^{1}(T)^{n}$ we have

$$
\Pi_{\hat{T}} \hat{\mathbf{v}}=\widehat{\Pi_{T} \mathbf{v}}
$$

Proof. We have to check that $\widehat{\Pi_{T} \mathbf{v}}$ satisfies the conditions defining $\Pi_{\widehat{T}} \hat{\mathbf{v}}$, namely,

$$
\int_{\widehat{F}_{i}} \widehat{\Pi_{T} \mathbf{v}} \cdot \hat{\mathbf{n}}_{i} \hat{p}_{k} d \hat{s}=\int_{\widehat{F}_{i}} \hat{\mathbf{v}} \cdot \hat{\mathbf{n}}_{i} \hat{p}_{k} d \hat{s} \quad \forall \hat{p}_{k} \in \mathcal{P}_{k}\left(\widehat{F}_{i}\right), \quad i=1, \cdots, n+1,
$$


where $\widehat{F}_{i}=F^{-1}\left(F_{i}\right)$, and

$$
\int_{\widehat{T}} \widehat{\Pi_{T} \mathbf{v}} \cdot \hat{\mathbf{p}}_{k-1} d \hat{x}=\int_{\widehat{T}} \hat{\mathbf{v}} \cdot \hat{\mathbf{p}}_{k-1} d \hat{x} \quad \forall \hat{\mathbf{p}}_{k-1} \in \mathcal{P}_{k-1}^{n}(\widehat{T}) .
$$

Given $\hat{p}_{k} \in \mathcal{P}_{k}\left(\widehat{F}_{i}\right)$ we have

$$
\int_{\widehat{F}_{i}} \hat{\mathbf{v}} \cdot \hat{\mathbf{n}}_{i} \hat{p}_{k} d \hat{s}=\int_{F_{i}} \mathbf{v} \cdot \mathbf{n}_{i} p_{k} d s .
$$

Indeed, this follows from (3.13) by a density argument. We can not apply (3.13) directly because the function obtained by extending $p_{k}$ by zero to the other faces of $T$ is not in $H^{\frac{1}{2}}(\partial T)$ and, therefore, it is not the restriction to the boundary of a function $\phi \in H^{1}(T)$. However, we can take a sequence of functions $q_{j} \in C_{0}^{\infty}\left(F_{i}\right)$ such that $q_{j} \rightarrow p_{k}$ in $L^{2}\left(F_{i}\right)$ and, since the extension by zero to $\partial T$ of $q_{j}$ is in $H^{\frac{1}{2}}(\partial T)$, there exists $\phi_{j} \in H^{1}(T)$ such that the restriction of $\phi_{j}$ to $F_{i}$ is equal to $q_{j}$. Therefore, applying (3.13) we obtain,

$$
\int_{\widehat{F}_{i}} \hat{\mathbf{v}} \cdot \hat{\mathbf{n}}_{i} \hat{q}_{j} d \hat{s}=\int_{F_{i}} \mathbf{v} \cdot \mathbf{n}_{i} q_{j} d s
$$

and therefore, since $\mathbf{v} \cdot \mathbf{n}_{i} \in L^{2}\left(F_{i}\right)$, we can pass to the limit to obtain (3.17). Analogously we have

$$
\int_{\widehat{F}_{i}} \widehat{\Pi_{T} \mathbf{v}} \cdot \hat{\mathbf{n}}_{i} \hat{p}_{k} d \hat{s}=\int_{F_{i}} \Pi_{T} \mathbf{v} \cdot \mathbf{n}_{i} p_{k} d s .
$$

and therefore (3.15) follows from condition (3.6) in the definition of $\Pi_{T} \mathbf{v}$.

To check (3.16) observe that, for $\hat{\mathbf{p}}_{k-1} \in \mathcal{P}_{k-1}^{n}(\widehat{T})$, we have

$$
\begin{gathered}
\int_{\widehat{T}} \widehat{\Pi_{T} \mathbf{v}} \cdot \hat{\mathbf{p}}_{k-1} d \hat{x}=\int_{T}|J| A^{-1} \Pi_{T} \mathbf{v} \cdot|J| A^{-1} \mathbf{p}_{k-1}|J|^{-1} d x \\
=\int_{T} \Pi_{T} \mathbf{v} \cdot|J| A^{-T} A^{-1} \mathbf{p}_{k-1} d x=\int_{T} \mathbf{v} \cdot|J| A^{-T} A^{-1} \mathbf{p}_{k-1} d x=\int_{\widehat{T}} \hat{\mathbf{v}} \cdot \hat{\mathbf{p}}_{k-1} d \hat{x}
\end{gathered}
$$

where we have used condition (3.7) and that $|J| A^{-T} A^{-1} \mathbf{p}_{k-1} \in \mathcal{P}_{k-1}^{n}(T)$.

We can now prove the optimal order error estimates for the RaviartThomas interpolation.

Theorem 3.5 There exists a constant $C$ depending on $k, n$ and the regularity constant $\sigma$ such that, for any $\mathbf{v} \in H^{m}(T)^{n}$ and $1 \leq m \leq k+1$,

$$
\left\|\mathbf{v}-\Pi_{T} \mathbf{v}\right\|_{L^{2}(T)} \leq C h_{T}^{m}\left\|\nabla^{m} \mathbf{v}\right\|_{L^{2}(T)} .
$$


Proof. First we prove an estimate on the reference element $\widehat{T}$. We will denote with $\widehat{C}$ a generic constant which depends only on $k, n$ and $\widehat{T}$. For each face $\widehat{F}_{i}$ of $\widehat{T}$ let $\left\{p_{j}^{i}\right\}_{1 \leq j \leq N}$ be a basis of $\mathcal{P}_{k}\left(\widehat{F}_{i}\right)$ and let $\left\{\mathbf{p}_{m}\right\}_{1 \leq m \leq M}$ be a basis of $\mathcal{P}_{k-1}^{n}(\widehat{T})$. Then, associated with this basis we can introduce the Lagrange-type basis of $\mathcal{R} \mathcal{T}_{k}(\widehat{T}),\left\{\phi_{j}^{i}, \psi_{m}\right\}$ defined by

$$
\begin{gathered}
\int_{\widehat{F}_{i}} \phi_{j}^{i} \cdot \mathbf{n}_{i} p_{s}^{r}=\delta_{i r} \delta_{j s} \quad, \quad \int_{\widehat{T}} \phi_{j}^{i} \cdot \mathbf{p}_{m}=0, \\
\forall \quad i, r=1, \cdots, n+1 \quad, \quad j, s=1, \cdots, N \quad, \quad m=1, \cdots, M
\end{gathered}
$$

and

$$
\begin{gathered}
\int_{\widehat{T}} \psi_{m} \cdot \mathbf{p}_{\ell}=\delta_{m \ell} \quad, \quad \psi_{m} \cdot \mathbf{n}_{i}=0 \\
\forall \quad m, \ell=1, \cdots, M \quad, \quad i=1, \cdots, n+1 .
\end{gathered}
$$

Then,

$$
\Pi_{\widehat{T}} \hat{\mathbf{v}}(\hat{x})=\sum_{i=1}^{n} \sum_{j=1}^{N}\left(\int_{\widehat{F}_{i}} \hat{\mathbf{v}} \cdot \mathbf{n}_{i} p_{j}^{i}\right) \phi_{j}^{i}(\hat{x})+\sum_{m=1}^{M}\left(\int_{\widehat{T}} \hat{\mathbf{v}} \cdot \mathbf{p}_{m}\right) \psi_{m}(\hat{x}) .
$$

Now, from the trace theorem (2.1) on $\widehat{T}$ we have

$$
\left|\int_{\widehat{F}_{i}} \hat{\mathbf{v}} \cdot \mathbf{n}_{i} p_{j}^{i}\right| \leq \widehat{C}\|\hat{\mathbf{v}}\|_{H^{1}(\widehat{T})} .
$$

Clearly, we also have

$$
\left|\int_{\hat{T}} \hat{\mathbf{v}} \cdot \mathbf{p}_{m}\right| \leq \widehat{C}\|\hat{\mathbf{v}}\|_{L^{2}(\widehat{T})}
$$

In both estimates the constant $\widehat{C}$ depends on bounds for the polynomials $p_{j}^{i}$ and $\mathbf{p}_{m}$ and then, it depends only on $k, n$ and $\widehat{T}$.

Therefore, using now that $\left\|\phi_{j}^{i}\right\|_{L^{2}(\widehat{T})}$ and $\left\|\psi_{m}\right\|_{L^{2}(\widehat{T})}$ are also bounded by a constant $\widehat{C}$ we obtain

$$
\left\|\Pi_{\widehat{T}} \hat{\mathbf{v}}\right\|_{L^{2}(\widehat{T})} \leq \widehat{C}\|\hat{\mathbf{v}}\|_{H^{1}(\widehat{T})} .
$$

Using now the relation (3.14) and making a change of variables we have

$$
\int_{T}\left|\Pi_{T} \mathbf{v}\right|^{2} d x=\int_{\widehat{T}}|J|^{-2}\left|A \Pi_{\widehat{T}} \hat{\mathbf{v}}\right|^{2}|J| d \hat{x} \leq|J|^{-1}\|A\|^{2} \int_{\widehat{T}}\left|\Pi_{\widehat{T}} \hat{\mathbf{v}}\right|^{2} d \hat{x}
$$


Then, using the bound for $\|A\|(2.10)$ and (3.19) we obtain

$$
\int_{T}\left|\Pi_{T} \mathbf{v}\right|^{2} d x \leq|J|^{-1} \frac{h_{T}^{2}}{\rho_{\widehat{T}}^{2}}\left\{\int_{\widehat{T}}|\hat{\mathbf{v}}|^{2} d \hat{x}+\int_{\widehat{T}}|\widehat{D} \hat{\mathbf{v}}|^{2} d \hat{x}\right\}
$$

but, since $\hat{\mathbf{v}}=|J| A^{-1} \mathbf{v}$ and $\widehat{D} \hat{\mathbf{v}}=|J| A^{-1} D \mathbf{v} A$, using the bounds for $\|A\|$ and $\left\|A^{-1}\right\|(2.10)$,

$$
|\hat{\mathbf{v}}| \leq|J| \frac{h_{\widehat{T}}}{\rho_{T}}|\mathbf{v}| \quad \text { and } \quad|\widehat{D} \hat{\mathbf{v}}| \leq|J| \frac{h_{\widehat{T}}}{\rho_{T}} \frac{h_{T}}{\rho_{\widehat{T}}}|D \mathbf{v}|
$$

and so, it follows from (3.20), changing variables again, that

$$
\left\|\Pi_{T} \mathbf{v}\right\|_{L^{2}(T)}^{2} \leq \widehat{C}\left\{\frac{h_{T}^{2}}{\rho_{T}^{2}}\|\mathbf{v}\|_{L^{2}(T)}^{2}+\frac{h_{T}^{4}}{\rho_{T}^{2}}\|D \mathbf{v}\|_{L^{2}(T)}^{2}\right\} .
$$

Therefore, from the regularity hypothesis (2.9) we obtain

$$
\left\|\Pi_{T} \mathbf{v}\right\|_{L^{2}(T)} \leq C\left\{\|\mathbf{v}\|_{L^{2}(T)}+h_{T}\|D \mathbf{v}\|_{L^{2}(T)}\right\}
$$

where the constant depends only on $\widehat{T}, k, n$ and the regularity constant $\sigma$.

Now we use a standard $\operatorname{argument.~Since~} \mathcal{P}_{k}^{n}(T) \subset \mathcal{R} \mathcal{T}(T)$ we know that $\Pi_{T} \mathbf{q}=\mathbf{q}$ for all $\mathbf{q} \in \mathcal{P}_{k}^{n}(T)$ and then

$\left\|\mathbf{v}-\Pi_{T} \mathbf{v}\right\|_{L^{2}(T)}=\left\|\mathbf{v}-\mathbf{q}-\Pi_{T}(\mathbf{v}-\mathbf{q})\right\|_{L^{2}(T)} \leq C\left\{\|\mathbf{v}-\mathbf{q}\|_{L^{2}(T)}+h_{T}\|D(\mathbf{v}-\mathbf{q})\|_{L^{2}(T)}\right\}$

where the constant depends on that in (3.21). Therefore, we conclude the proof applying Lemma 2.2 .

Let us now introduce the global Raviart-Thomas finite element spaces. Assume that we have a family of triangulations $\left\{\mathcal{T}_{h}\right\}$ of $\Omega$, i.e., $\Omega=\cup_{T \in \mathcal{T}_{h}} T$, such that the intersection of two triangles in $\mathcal{T}_{h}$ is either empty, or a vertex, or a common side and $h$ is a measure of the mesh-size, namely, $h=\max _{T \in \mathcal{T}_{h}} h_{T}$.

We assume that the family of triangulations is regular, i.e., for any $T \in$ $\mathcal{T}_{h}$ and any $h$, the regularity condition (2.9) is satisfied with a uniform $\sigma$.

Associated with the triangulation $\mathcal{T}_{h}$ we introduce the global space

$$
\mathcal{R} \mathcal{T}_{k}\left(\mathcal{T}_{h}\right)=\left\{\mathbf{v} \in H(\operatorname{div}, \Omega):\left.\mathbf{v}\right|_{T} \in \mathcal{R} \mathcal{T}_{k}(T) \quad \forall T \in \mathcal{T}_{h}\right\}
$$

When no confusion arises we will drop the $\mathcal{T}_{h}$ from the definition and call $\mathcal{R} \mathcal{T}_{k}$ the global space. A fundamental tool in the error analysis is the operator

$$
\Pi_{h}: H(\operatorname{div}, \Omega) \cap \prod_{T \in \mathcal{T}_{h}} H^{1}(T)^{n} \longrightarrow \mathcal{R} \mathcal{T}_{k}
$$


defined by

$$
\left.\Pi_{h} \mathbf{v}\right|_{T}=\Pi_{T} \mathbf{v} \quad \forall T \in \mathcal{T}_{h}
$$

We have to check that $\Pi_{h} \mathbf{v} \in \mathcal{R} \mathcal{T}_{k}$. Since by definition $\Pi_{T} \mathbf{v} \in \mathcal{R} \mathcal{T}_{k}(T)$, it only remains to see that $\Pi_{h} \mathbf{v} \in H(\operatorname{div}, \Omega)$.

First we observe that a piecewise polynomial vector function is in $H(\operatorname{div}, \Omega)$ if and only if it has continuous normal component across the elements (this can be verified by applying the divergence theorem). But, since $\mathbf{v} \in$ $H(\operatorname{div}, \Omega)$, the continuity of the normal component of $\Pi_{h} \mathbf{v}$ follows from $b$ ) of Lemma 3.1 in view of the degrees of freedom (3.6) in the definition of $\Pi_{T}$.

The finite element space for the approximation of the scalar variable $p$ is the standard space of, not necessarily continuous, piecewise polynomials of degree $k$, namely,

$$
\mathcal{P}_{k}^{d}\left(\mathcal{T}_{h}\right)=\left\{q \in L^{2}(\Omega):\left.q\right|_{T} \in \mathcal{P}_{k}(T): \quad \forall T \in \mathcal{T}_{h}\right\}
$$

where the $d$ stands for "discontinuous". Also in this case we will write only $\mathcal{P}_{k}^{d}$ when no confusion arises. Observe that, since no derivative of the scalar variable appears in the weak form, we do not require any continuity in the approximation space for this variable.

In the following lemma we give two fundamental properties for the error analysis.

Lemma 3.6 The operator $\Pi_{h}$ satisfies

$$
\int_{\Omega} \operatorname{div}\left(\mathbf{v}-\Pi_{h} \mathbf{v}\right) q d x=0
$$

$\forall \mathbf{v} \in H(\operatorname{div}, \Omega) \cap \prod_{T \in \mathcal{T}_{h}} H^{1}(T)^{n}$ and $\forall q \in \mathcal{P}_{k}^{d}$. Moreover,

$$
\operatorname{div} \mathcal{R} \mathcal{T}_{k}=\mathcal{P}_{k}^{d}
$$

Proof. Using (3.6) and (3.7) it follows that, for any $\mathbf{v} \in H^{1}(T)^{n}$ and any $q \in \mathcal{P}_{k}(T)$,

$$
\int_{T} \operatorname{div}\left(\mathbf{v}-\Pi_{T} \mathbf{v}\right) q d x=-\int_{T}\left(\mathbf{v}-\Pi_{T} \mathbf{v}\right) \cdot \nabla q d x+\int_{\partial T}\left(\mathbf{v}-\Pi_{T} \mathbf{v}\right) \cdot \mathbf{n} q=0
$$

thus, (3.24) holds.

It is easy to see that $\operatorname{div} \mathcal{R} \mathcal{T}_{k} \subset \mathcal{P}_{k}^{d}$. In order to see the other inclusion recall that from Lemma 2.4 we know that $\operatorname{div}: H^{1}(\Omega)^{n} \rightarrow L^{2}(\Omega)$ is surjective. Therefore, given $q \in \mathcal{P}_{k}^{d}$ there exists $\mathbf{v} \in H^{1}(\Omega)^{n}$ such that $\operatorname{div} \mathbf{v}=q$. Then, it follows from (3.24) that $\operatorname{div} \Pi_{h} \mathbf{v}=q$ and so (3.25) is proved. 
Introducing the orthogonal $L^{2}$-projection $P_{h}: L^{2}(\Omega) \rightarrow \mathcal{P}_{k}^{d}$, properties (3.24) and (3.25) can be summarized in the following commutative diagram

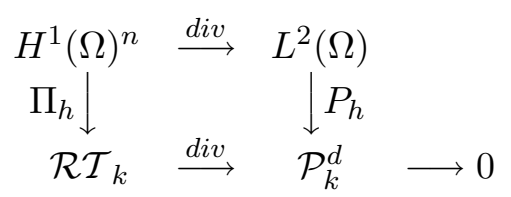

where, to simplify notation, we have replaced $H(\operatorname{div}, \Omega) \cap \prod_{T \in \mathcal{T}_{h}} H^{1}(T)^{n}$ by its subspace $H^{1}(\Omega)^{n}$.

Our next goal is to give error estimates for the mixed finite element approximation of Problem 3.1, namely, $\left(\mathbf{u}_{h}, p_{h}\right) \in \mathcal{R} \mathcal{T}_{k} \times \mathcal{P}_{k}^{d}$ defined by

$$
\left\{\begin{aligned}
\int_{\Omega} \mu \mathbf{u}_{h} \cdot \mathbf{v} d x-\int_{\Omega} p_{h} \operatorname{div} \mathbf{v} d x=0 & \forall \mathbf{v} \in \mathcal{R} \mathcal{T}_{k} \\
\int_{\Omega} q \operatorname{div} \mathbf{u}_{h} d x=\int_{\Omega} f q d x & \forall q \in \mathcal{P}_{k}^{d}
\end{aligned}\right.
$$

It is important to remark that, although we are considering the particular case of the Raviart-Thomas spaces on simplicial elements, the error analysis only makes use of the fundamental commutative diagram property (3.26) and of the approximation properties of the projections $\Pi_{h}$ and $P_{h}$. Therefore, similar results can be obtained for other finite element spaces.

Lemma 3.7 If $\mathbf{u}$ and $\mathbf{u}_{h}$ are the solutions of (3.3) and (3.27) then,

$$
\left\|\mathbf{u}-\mathbf{u}_{h}\right\|_{L^{2}(\Omega)} \leq\left(1+\|a\|_{L^{\infty}(\Omega)}\|\mu\|_{L^{\infty}(\Omega)}\right)\left\|\mathbf{u}_{h}-\Pi_{h} \mathbf{u}\right\|_{L^{2}(\Omega)}
$$

Proof. Subtracting (3.27) from (3.3) we obtain the error equations

$$
\int_{\Omega} \mu\left(\mathbf{u}-\mathbf{u}_{h}\right) \cdot \mathbf{v} d x-\int_{\Omega}\left(p-p_{h}\right) \operatorname{div} \mathbf{v} d x=0 \quad \forall \mathbf{v} \in \mathcal{R} \mathcal{T}_{k}
$$

and,

$$
\int_{\Omega} q \operatorname{div}\left(\mathbf{u}-\mathbf{u}_{h}\right) d x=0 \quad \forall q \in \mathcal{P}_{k}^{d}
$$

Using (3.24) and (3.29) we obtain

$$
\int_{\Omega} q \operatorname{div}\left(\Pi_{h} \mathbf{u}-\mathbf{u}_{h}\right) d x=0 \quad \forall q \in \mathcal{P}_{k}^{d}
$$


and, since (3.25) holds, we can take $q=\operatorname{div}\left(\Pi_{h} \mathbf{u}-\mathbf{u}_{h}\right)$ to conclude that

$$
\operatorname{div}\left(\Pi_{h} \mathbf{u}-\mathbf{u}_{h}\right)=0 .
$$

Therefore, taking $\mathbf{v}=\Pi_{h} \mathbf{u}-\mathbf{u}_{h}$ in (3.28) we obtain

$$
\int_{\Omega} \mu\left(\mathbf{u}-\mathbf{u}_{h}\right) \cdot\left(\Pi_{h} \mathbf{u}-\mathbf{u}_{h}\right) d x=0
$$

and so,

$$
\begin{gathered}
\left\|\Pi_{h} \mathbf{u}-\mathbf{u}_{h}\right\|_{L^{2}(\Omega)}^{2} \leq\|a\|_{L^{\infty}(\Omega)} \int_{\Omega} \mu\left(\Pi_{h} \mathbf{u}-\mathbf{u}\right)\left(\Pi_{h} \mathbf{u}-\mathbf{u}_{h}\right) d x \\
\quad \leq\|a\|_{L^{\infty}(\Omega)}\|\mu\|_{L^{\infty}(\Omega)}\left\|\Pi_{h} \mathbf{u}-\mathbf{u}\right\|_{L^{2}(\Omega)}\left\|\Pi_{h} \mathbf{u}-\mathbf{u}_{h}\right\|_{L^{2}(\Omega)}
\end{gathered}
$$

and we conclude the proof by using the triangle inequality. $\square$

As a consequence, we have the following optimal order error estimate for the approximation of the vector variable $\mathbf{u}$.

Theorem 3.8 If the solution $\mathbf{u}$ of Problem 3.2 belongs to $H^{m}(\Omega)^{n}, 1 \leq$ $m \leq k+1$, there exists a constant $C$ depending on $\|a\|_{L^{\infty}(\Omega)},\|\mu\|_{L^{\infty}(\Omega)}, k$, $n$ and the regularity constant $\sigma$, such that

$$
\left\|\mathbf{u}-\mathbf{u}_{h}\right\|_{L^{2}(\Omega)} \leq C h^{m}\left\|\nabla^{m} \mathbf{u}\right\|_{L^{2}(\Omega)}
$$

Proof. The result is an immediate consequence of Lemma 3.7 and Theorem 3.5.

In the next theorem we obtain error estimates for the scalar variable $p$. We will use that

$$
\left\|\mathbf{v}-\Pi_{h} \mathbf{v}\right\|_{L^{2}(\Omega)} \leq C h\|\mathbf{v}\|_{H^{1}(\Omega)} \forall \mathbf{v} \in H^{1}(\Omega)
$$

which follows from a particular case of Theorem 3.5. In particular,

$$
\left\|\Pi_{h} \mathbf{v}\right\|_{L^{2}(\Omega)} \leq C\|\mathbf{v}\|_{H^{1}(\Omega)} .
$$

Lemma 3.9 If $(\mathbf{u}, p)$ and $\left(\mathbf{u}_{h}, p_{h}\right)$ are the solutions of (3.3) and (3.27), there exists a constant $C$ depending on $\|a\|_{L^{\infty}(\Omega)},\|\mu\|_{L^{\infty}(\Omega)}, k, n, \Omega$ and the regularity constant $\sigma$, such that

$$
\left\|p-p_{h}\right\|_{L^{2}(\Omega)} \leq C\left\{\left\|p-P_{h} p\right\|_{L^{2}(\Omega)}+\left\|\mathbf{u}-\Pi_{h} \mathbf{u}\right\|_{L^{2}(\Omega)}\right\}
$$


Proof. From (3.25) we know that for any $q \in \mathcal{P}_{k}^{d}$ there exists $\mathbf{w}_{h} \in \mathcal{R} \mathcal{T}_{k}$ such that $\operatorname{div} \mathbf{w}_{h}=q$. Moreover, it is easy to see that $\mathbf{w}_{h}$ can be taken such that

$$
\left\|\mathbf{w}_{h}\right\|_{L^{2}(\Omega)} \leq C\|q\|_{L^{2}(\Omega)} .
$$

Indeed, recall that $\mathbf{w}_{h}=\Pi_{h} \mathbf{w}$ where $\mathbf{w} \in H^{1}(\Omega)$ satisfies $\operatorname{div} \mathbf{w}=q$ and $\|\mathbf{w}\|_{H^{1}(\Omega)} \leq C\|q\|_{L^{2}(\Omega)}$ (from Lemma 2.4 we know that such a w exists). Then, (3.33) follows from (3.31).

Now, from the error equation (3.28) we have

$$
\int_{\Omega}\left(P_{h} p-p_{h}\right) \operatorname{div} \mathbf{v} d x=\int_{\Omega}\left(\mathbf{u}-\mathbf{u}_{h}\right) \mathbf{v} d x \quad \forall \mathbf{v} \in \mathcal{R} \mathcal{T}_{k}
$$

and so, taking $\mathbf{v} \in V_{h}$ such that $\operatorname{div} \mathbf{v}=P_{h} p-p_{h}$ and

$$
\|\mathbf{v}\|_{L^{2}(\Omega)} \leq C\left\|P_{h} p-p_{h}\right\|_{L^{2}(\Omega)},
$$

we obtain

$$
\left\|P_{h} p-p_{h}\right\|_{L^{2}(\Omega)}^{2} \leq C\left\|\mathbf{u}-\mathbf{u}_{h}\right\|_{L^{2}(\Omega)}\left\|P_{h} p-p_{h}\right\|_{L^{2}(\Omega)}
$$

which combined with Lemma 3.7 and the triangular inequality yields (3.32). $\square$

As a consequence, we obtain an error estimate for the approximation of the scalar variable $p$.

Theorem 3.10 If the solution $(\mathbf{u}, p)$ of Problem 3.2 belongs to $H^{m}(\Omega)^{n} \times$ $H^{m}(\Omega), 1 \leq m \leq k+1$, there exists a constant $C$ depending on $\|a\|_{L^{\infty}(\Omega)}$, $\|\mu\|_{L^{\infty}(\Omega)}, k, n$ and the regularity constant $\sigma$, such that

$$
\left\|p-p_{h}\right\|_{L^{2}(\Omega)} \leq C h^{m}\left\{\left\|\nabla^{m} \mathbf{u}\right\|_{L^{2}(\Omega)}+\left\|\nabla^{m} p\right\|_{L^{2}(\Omega)}\right\}
$$

Proof. The result follows immediately from Theorem 3.8, Lemma 3.9 and the error estimates for the $L^{2}$-projection given in (2.3).

For the case in which $\Omega$ is a convex polygon or a smooth domain and the coefficient $a$ is smooth enough to have the a priori estimate

$$
\|p\|_{H^{2}(\Omega)} \leq C_{0}\|f\|_{L^{2}(\Omega)}
$$

we also obtain a higher order error estimate for $\left\|P_{h} p-p_{h}\right\|_{L^{2}(\Omega)}$ using a duality argument. 
Lemma 3.11 If $a \in W^{1, \infty}(\Omega)$ and (3.35) holds, there exists a constant $C$ depending on $\|a\|_{W^{1, \infty}(\Omega)},\|\mu\|_{L^{\infty}(\Omega)}, k, n, \Omega$ and $C_{0}$ such that

$$
\left\|P_{h} p-p_{h}\right\|_{L^{2}(\Omega)} \leq C h\left\{\left\|\mathbf{u}-\mathbf{u}_{h}\right\|_{L^{2}(\Omega)}+\left\|\operatorname{div}\left(\mathbf{u}-\mathbf{u}_{h}\right)\right\|_{L^{2}(\Omega)}\right\}
$$

Proof. We use a duality argument. Let $\phi$ be the solution of

$$
\left\{\begin{aligned}
\operatorname{div}(a \nabla \phi) & =P_{h} p-p_{h} & & \text { in } \Omega \\
\phi & =0 & & \text { on } \partial \Omega
\end{aligned}\right.
$$

Using (3.24), (3.25), (3.28), (3.29), and (3.30) we have,

$$
\begin{aligned}
& \left\|P_{h} p-p_{h}\right\|_{L^{2}(\Omega)}^{2}=\int_{\Omega}\left(P_{h} p-p_{h}\right) \operatorname{div}(a \nabla \phi) d x=\int_{\Omega}\left(P_{h} p-p_{h}\right) \operatorname{div} \Pi_{h}(a \nabla \phi) d x \\
& =\int_{\Omega}\left(p-p_{h}\right) \operatorname{div} \Pi_{h}(a \nabla \phi) d x=\int_{\Omega} \mu\left(\mathbf{u}-\mathbf{u}_{h}\right) \cdot\left(\Pi_{h}(a \nabla \phi)-a \nabla \phi\right) d x \\
& +\int_{\Omega}\left(\mathbf{u}-\mathbf{u}_{h}\right) \cdot \nabla \phi d x=\int_{\Omega} \mu\left(\mathbf{u}-\mathbf{u}_{h}\right) \cdot\left(\Pi_{h}(a \nabla \phi)-a \nabla \phi\right) d x-\int_{\Omega} \operatorname{div}\left(\mathbf{u}-\mathbf{u}_{h}\right)\left(\phi-P_{h} \phi\right) d x \\
& \quad \leq C\left\|\mathbf{u}-\mathbf{u}_{h}\right\|_{L^{2}(\Omega)} h\|\phi\|_{H^{2}(\Omega)}+C\left\|\operatorname{div}\left(\mathbf{u}-\mathbf{u}_{h}\right)\right\|_{L^{2}(\Omega)} h\|\phi\|_{H^{1}(\Omega)}
\end{aligned}
$$

where for the last inequality we have used that $a \in W^{1, \infty}(\Omega)$. The proof concludes by using the a priori estimate (3.35) for $\phi$.

Theorem 3.12 If $a \in W^{1, \infty}(\Omega)$, (3.35) holds, $\mathbf{u} \in H^{k+1}(\Omega)^{n}$ and $f \in$ $H^{k+1}(\Omega)$, there exists a constant $C$ depending on $\|a\|_{W^{1, \infty}(\Omega)},\|\mu\|_{L^{\infty}(\Omega)}, k$, $n, \Omega$ and $C_{0}$ such that

$$
\left\|P_{h} p-p_{h}\right\|_{L^{2}(\Omega)} \leq C h^{k+2}\left\{\left\|\nabla^{k+1} \mathbf{u}\right\|_{L^{2}(\Omega)}+\left\|\nabla^{k+1} f\right\|_{L^{2}(\Omega)}\right\}
$$

Proof. The second equation in (3.27) can be written as $\operatorname{div} \mathbf{u}_{h}=P_{h} f$. Then we have

$$
\operatorname{div}\left(\mathbf{u}-\mathbf{u}_{h}\right)=f-P_{h} f
$$

and, therefore, the theorem follows from Theorem 3.8 and Lemma 3.11 and the error estimates for the $L^{2}$-projection given in (2.3).

The estimate for $\left\|P_{h} p-p_{h}\right\|_{L^{2}(\Omega)}$ given by this theorem is important because it can be used to construct superconvergent approximations of $p$, i.e., 
approximations which converge at a higher order than $p_{h}$ (see for example $[11,48])$

For the sake of clarity we have presented the error analysis for the Raviart-Thomas spaces which were the first ones introduced for the mixed approximation of second order elliptic problems. However, as we mentioned above, the analysis makes use only of the existence of a projection $\Pi_{h}$ satisfying the commutative diagram property and on approximation properties of $\Pi_{h}$ and of the $L^{2}$-projection on the finite element space used to approximate the scalar variable $p$.

For the particular case of the Raviart-Thomas spaces the regularity assumption (2.9) can be replaced by the weaker "maximum angle condition" (see [1] for $k=0$ and $n=2,3$, [28] for $k=1$ and $n=2$ and [29] for general $k \geq 0$ and $n=2$ ).

The Raviart-Thomas spaces were constructed in order to approximate both vector and scalar variables with the same order. However, if one is more interested in the approximation of the vector variable $\mathbf{u}$, one can try to use different order approximations for each variable in order to reduce the degrees of freedom (thus reducing the computational cost) while preserving the same order of convergence for $\mathbf{u}$ provided by the $\mathcal{R} \mathcal{T}_{k}$ spaces. This is the main idea to define the following spaces which were introduced by Brezzi, Douglas and Marini [16]. Although with this choice the order of convergence for $p$ is reduced, estimate (3.37) allows to improve it by a post-processing of the computed solution [16].

In the examples below, we will define the local spaces for each variable. It is not difficult to check that the degrees of freedom defining the spaces approximating the vector variable guarantee the continuity of the normal component and therefore the global spaces are subspaces of $H(\operatorname{div}, \Omega)$.

For $n=2, k \geq 1$ and $T$ a triangle, the space $\mathcal{B D}_{\mathcal{B}}(T)$ is defined in the following way:

$$
\mathcal{B D}_{k}(T)=\mathcal{P}_{k}^{2}(T)
$$

and the corresponding space for the scalar variable is $\mathcal{P}_{k-1}(T)$.

Observe that

$$
\operatorname{dim} \mathcal{B D}_{k}(T)=(k+1)(k+2) .
$$

For example, $\operatorname{dim} \mathcal{B D}_{1}(T)=6$ and $\operatorname{dim} \mathcal{B D}_{1} \mathcal{M}_{2}(T)=12$. Figure 3 shows the degrees of freedom for these two spaces. The arrows correspond to degrees of freedom of normal components while the circles indicate the internal 

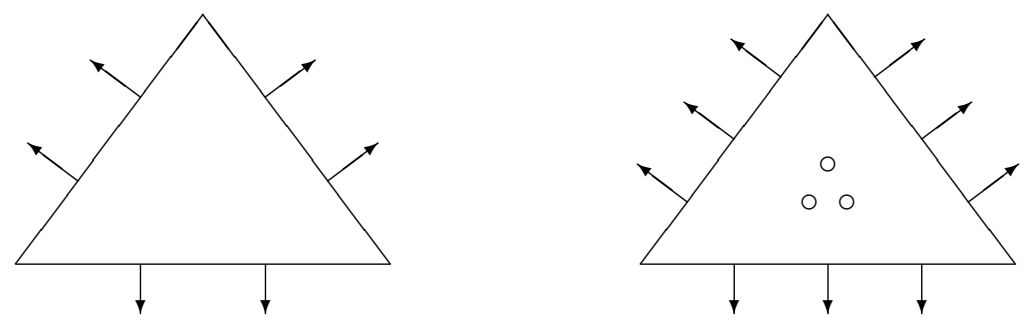

Figure 3: Degrees of freedom for $\mathcal{B D}_{1}$ and $\mathcal{B D}_{2}$

degrees of freedom corresponding to the second and third conditions in the definition of $\Pi_{T}$ below.

In what follows, $\ell_{i}, i=1,2,3$ are the sides of $T, b_{T}=\lambda_{1} \lambda_{2} \lambda_{3}$ is a "bubble" function and, for $\phi \in H^{1}(\Omega)$,

$$
\operatorname{curl} \phi=\left(\frac{\partial \phi}{\partial y},-\frac{\partial \phi}{\partial x}\right)
$$

The operator $\Pi_{T}$ for this case is defined as follows:

$$
\begin{gathered}
\int_{\ell_{i}} \Pi_{T} \mathbf{v} \cdot \mathbf{n}_{i} p_{k} d s=\int_{\ell_{i}} \mathbf{v} \cdot \mathbf{n}_{i} p_{k} d s \quad \forall p_{k} \in \mathcal{P}_{k}\left(\ell_{i}\right), \quad i=1,2,3 \\
\int_{T} \Pi_{T} \mathbf{v} \cdot \nabla p_{k-1} d x=\int_{T} \mathbf{v} \cdot \nabla p_{k-1} d x \quad \forall p_{k-1} \in \mathcal{P}_{k-1}(T)
\end{gathered}
$$

and, when $k \geq 2$

$$
\int_{T} \Pi_{T} \mathbf{v} \cdot \operatorname{curl}\left(b_{T} p_{k-2}\right) d x=\int_{T} \mathbf{v} \cdot \operatorname{curl}\left(b_{T} p_{k-2}\right) d x \quad \forall p_{k-2} \in \mathcal{P}_{k-2}(T)
$$

The reader can check that all the conditions for convergence are satisfied in this case. Property (3.24) follows from the definition of $\Pi_{T}$ and the proof of its existence is similar to that of Lemma 3.2. Consequently, the same arguments used for the Raviart-Thomas approximation provide the same error estimate for the approximation of $\mathbf{u}$ that we had in Theorem 3.8 while for $p$ we have

$$
\left\|p-p_{h}\right\|_{L^{2}(\Omega)} \leq C h^{m}\left\{\left\|\nabla^{m} \mathbf{u}\right\|_{L^{2}(\Omega)}+\left\|\nabla^{m} p\right\|_{L^{2}(\Omega)}\right\},
$$


$1 \leq m \leq k$ and the estimate does not hold for $m=k+1$ i.e., the best order of convergence is reduced in one with respect to the estimate obtained for the Raviart-Thomas approximation.

However, with the same argument used in Lemma 3.36 it can be proved that, for $k \geq 2$,

$$
\left\|P_{h} p-p_{h}\right\|_{L^{2}(\Omega)} \leq C\left\{h\left\|\mathbf{u}-\mathbf{u}_{h}\right\|_{L^{2}(\Omega)}+h^{2}\left\|\operatorname{div}\left(\mathbf{u}-\mathbf{u}_{h}\right)\right\|_{L^{2}(\Omega)}\right\},
$$

indeed, since $P_{h}$ is the orthogonal projection on $\mathcal{P}_{k-1}^{d}$ and $k-1 \geq 1$, this follows by using that

$$
\left\|\phi-P_{h} \phi\right\|_{L^{2}(\Omega)} \leq C h^{2}\|\phi\|_{H^{2}(\Omega)}
$$

in the last step of the proof of that lemma.

Therefore, for $k \geq 2$, we obtain the following result analogous to that in Theorem 3.37

$$
\left\|P_{h} p-p_{h}\right\|_{L^{2}(\Omega)} \leq C h^{k+2}\left\{\left\|\nabla^{k+1} \mathbf{u}\right\|_{L^{2}(\Omega)}+\left\|\nabla^{k} f\right\|_{L^{2}(\Omega)}\right\} .
$$

On the other hand, if $k=1,(3.39)$ does not hold (because in this case $P_{h}$ is the projection over piece-wise constant functions). Then, in this case we can prove only

$$
\left\|P_{h} p-p_{h}\right\|_{L^{2}(\Omega)} \leq C h^{2}\left\{\|\nabla \mathbf{u}\|_{L^{2}(\Omega)}+\|\nabla f\|_{L^{2}(\Omega)}\right\} .
$$

As we mentioned before, these estimates for $\left\|P_{h} p-p_{h}\right\|_{L^{2}(\Omega)}$ can be used to improve the order of approximation for $p$ by a local post-processing.

Several rectangular elements have also been introduced for mixed approximations. We recall some of them (and refer to [17] for a more complete review).

First we define the spaces introduced by Raviart and Thomas [44]. For nonnegative integers $j, k$ we call $\mathcal{Q}_{k, m}$ the space of polynomials of the form

$$
q(x, y)=\sum_{i=0}^{k} \sum_{j=0}^{m} a_{i j} x^{i} y^{j}
$$

then, the $\mathcal{R} \mathcal{T}_{k}(R)$ space on a rectangle $R$ is given by

$$
\mathcal{R} \mathcal{T}_{k}(R)=\mathcal{Q}_{k+1, k}(R) \times \mathcal{Q}_{k, k+1}(R)
$$




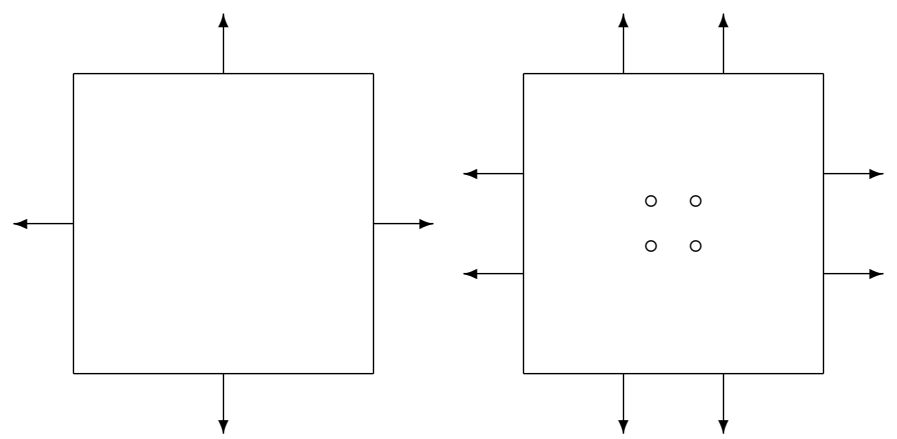

Figure 4: Degrees of freedom for $\mathcal{R} \mathcal{T}_{0}$ and $\mathcal{R} \mathcal{T}_{1}$

and the space for the scalar variable is $\mathcal{Q}_{k}(R)$. It can be easily checked that

$$
\operatorname{dim} \mathcal{R} \mathcal{T}_{k}(R)=2(k+1)(k+2) .
$$

Figure 4 shows the degrees of freedom for $k=0$ and $k=1$.

Denoting with $\ell_{i}, i=1,2,3,4$ the four sides of $R$, the degrees of freedom defining the operator $\Pi_{T}$ for this case are

$$
\int_{\ell_{i}} \Pi_{T} \mathbf{v} \cdot \mathbf{n}_{i} p_{k} d \ell=\int_{\ell_{i}} \mathbf{v} \cdot \mathbf{n}_{i} p_{k} d \ell \quad \forall p_{k} \in \mathcal{P}_{k}\left(\ell_{i}\right), \quad i=1,2,3,4
$$

and (for $k \geq 1$ )

$$
\int_{R} \Pi_{T} \mathbf{v} \cdot \phi_{k} d x=\int_{R} \mathbf{v} \cdot \phi_{k} d x \quad \forall \phi_{k} \in \mathcal{Q}_{k-1, k}(R) \times \mathcal{Q}_{k, k-1}(R)
$$

Our last example in the 2-d case are the spaces introduced by Brezzi, Douglas and Marini on rectangular elements. They are defined for $k \geq 1$ as

$$
\mathcal{B D}_{\mathcal{L}}(R)=\mathcal{P}_{k}^{2}(R)+\left\langle\operatorname{curl}\left(x^{k+1} y\right)\right\rangle+\left\langle\operatorname{curl}\left(x y^{k+1}\right)\right\rangle
$$

and the associated scalar space is $\mathcal{P}_{k-1}(R)$. It is easy to see that

$$
\operatorname{dim} \mathcal{B D}_{k}(R)=(k+1)(k+2)+2 .
$$

. The degrees of freedom for $k=1$ and $k=2$ are shown in Figure 5.

The operator $\Pi_{T}$ is defined by

$$
\int_{\ell_{i}} \Pi_{T} \mathbf{v} \cdot \mathbf{n}_{i} p_{k} d \ell=\int_{\ell_{i}} \mathbf{v} \cdot \mathbf{n}_{i} p_{k} d \ell \quad \forall p_{k} \in \mathcal{P}_{k}\left(\ell_{i}\right), \quad i=1,2,3,4
$$




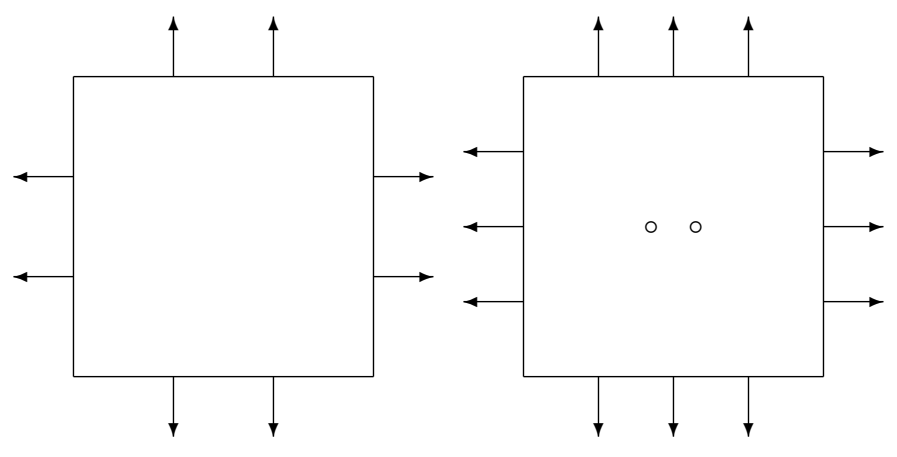

Figure 5: Degrees of freedom for $\mathcal{B D} \mathcal{M}_{1}$ and $\mathcal{B D} \mathcal{M}_{2}$

and (for $k \geq 2$ )

$$
\int_{R} \Pi_{T} \mathbf{v} \cdot \mathbf{p}_{k-2} d x=\int_{R} \mathbf{v} \cdot \mathbf{p}_{k-2} d x \quad \forall \mathbf{p}_{k-2} \in \mathcal{P}_{k-2}^{2}(R)
$$

The $\mathcal{R} \mathcal{T}_{k}$ as well as the $\mathcal{B D} \mathcal{M}_{k}$ spaces on rectangles have analogous properties to those on triangles. Therefore, the same error estimates obtained for triangular elements are valid in both cases.

More generally, one can consider general quadrilateral elements. Given a convex quadrilateral $Q$, the spaces are defined using the Piola transform from a reference rectangle $R$ to $Q$. Let us define for example the RaviartThomas spaces $\mathcal{R} \mathcal{T}_{k}(Q)$.

Let $R=[0,1] \times[0,1]$ be the reference rectangle and $F: R \rightarrow Q$ a bilinear transformation taking the vertices of $R$ into the vertices of $Q$. Then, we define the local space $\mathcal{R} \mathcal{T}_{k}(R)$ by using the Piola transform, i.e., if $x=F(\hat{x})$, $D F$ is the Jacobian matrix of $F$ and $J=|\operatorname{det} D F|$,

$$
\mathcal{R} \mathcal{T}_{k}(Q)=\left\{\mathbf{v}: Q \rightarrow \mathbb{R}^{2}: \mathbf{v}(x)=\frac{1}{J(\hat{x})} D F(\hat{x}) \hat{\mathbf{v}}(\hat{x}) \quad \text { with } \quad \hat{\mathbf{v}} \in \mathcal{R} \mathcal{T}_{k}(R)\right\}
$$

Also in this case similar error estimates to those obtained for triangular elements can be proved under appropriate regularity assumptions on the quadrilaterals. The analysis of this case is more technical and so we omit details and refer to $[5,37,49]$.

3-d extensions of the spaces defined above have been introduced by Nedelec $[41,42]$ and by Brezzi, Douglas, Durán and Fortin [14]. For tetrahedral 
elements the spaces are defined in an analogous way, although the construction of the operator $\Pi_{T}$ requires a different analysis (we refer to [41] for the extension of the $\mathcal{R} \mathcal{T}_{k}$ spaces and to $[42,14]$ for the extension of the $\mathcal{B D} \mathcal{M}_{k}$ spaces). In the case of 3 - $d$ rectangular elements, the extensions of $\mathcal{R} \mathcal{T}_{k}$ are again defined in an analogous way [41] and the extensions of $\mathcal{B D}_{\mathcal{k}}$ [14] can be defined for a 3 -d rectangle $R$ by

$$
\begin{aligned}
\mathcal{B D D F}_{k}(R)=\mathcal{P}_{k}^{3}+\left\langle\left\{\operatorname{curl}\left(0,0, x y^{i+1} z^{k-i}\right), \quad i=0, \ldots, k\right\}\right\rangle \\
+\left\langle\left\{\operatorname{curl}\left(0, x^{k-i} y z^{i+1}, 0\right), \quad i=0, \ldots, k\right\}\right\rangle \\
+\left\langle\left\{\operatorname{curl}\left(x^{i+1} y^{k-i} z, 0,0\right), \quad i=0, \ldots, k\right\}\right\rangle
\end{aligned}
$$

where now we are using the usual notation curl $\mathbf{v}$ for the rotational of a three dimensional vector field $\mathbf{v}$.

All the convergence results obtained in 2-d can be extended for the 3 -d spaces mentioned here. Other families of spaces, in both 2 and 3 dimensions which are intermediate between the $\mathcal{R} \mathcal{T}$ and the $\mathcal{B D} \mathcal{M}$ spaces were introduced and analyzed by Brezzi, Douglas, Fortin and Marini [15].

Finally, we refer to [10] for the case of general isoparametric hexahedral elements.

\section{A posteriori error estimates}

In this section we present an a posteriori error analysis for the mixed finite element approximation of second order elliptic problems. For simplicity, we will assume that the restriction of the coefficient $a$ in (3.1) to any element of the triangulation is constant. If not, higher order terms corresponding to the approximation of $a$ arise in the estimates.

For simplicity, we prove the results for the approximations obtained by the Raviart-Thomas spaces and in the two dimensional case. However, simple variants of the method can be applied for mixed approximations in other spaces, in particular, for all the spaces described in the previous section.

We introduce error estimators of the residual type for both scalar and vector variables and prove that the error is bounded by a constant times the estimator plus a term which is of higher order (i.e., what is usually called "reliability" of the estimator). We also prove that the estimator is less than or equal a constant times the error. This last estimate (usually called "efficiency" of the estimator) is local, more precisely, the error in one 
element $T$ can be bounded below by the estimators in the same triangle plus the estimators in the elements sharing a side with $T$.

It is well known that several mixed methods are related to non-conforming finite element approximations (see [6]). In particular the lowest order RaviartThomas method corresponds to the non-conforming linear elements of CrouzeixRaviart (see also [40]).

A posteriori error estimates were obtained first for the Crouzeix-Raviart method by using a Helmoltz type decomposition of the error (see [23]). The same technique has been applied for mixed finite element approximations in $[4,18]$. In [4] only the vector variable is estimated while in [18] both variables are estimated, but to estimate the scalar variable the a priori estimate (3.35) was assumed to hold. In particular, this hypothesis excludes non-convex polygonal domains. We refer also to $[3,39]$ for related results.

Our analysis for the vector variable follows the approach of $[4,18]$, while for the scalar variable we present a new argument which does not require the a priori estimate (3.35).

We will use the following well-known approximation result. We denote with $\mathcal{P}_{k+1}^{c}$ the standard continuous piece-wise polynomials of degree $k+1$. For any $\phi \in H^{1}(\Omega)$ there exists $\phi_{h} \in \mathcal{P}_{k+1}^{c}$ such that

$$
\left\|\phi-\phi_{h}\right\|_{0, \ell} \leq C|\ell|^{1 / 2}\|\nabla \phi\|_{L^{2}(\widetilde{T})}
$$

and,

$$
\left\|\phi-\phi_{h}\right\|_{0, T} \leq C|T|^{1 / 2}\|\nabla \phi\|_{L^{2}(\widetilde{T})}
$$

where $\widetilde{T}$ is the union of all the elements sharing a vertex with $T$ (we can take for example the Clément approximation [21] or any variant of it (see for example $[37,47])$.

We will use the notation curl $\phi$ introduced in the previous section for $\phi \in H^{1}(\Omega)$ and for $\mathbf{v} \in H^{1}(\Omega)^{2}$ we define

$$
\operatorname{rot} \mathbf{v}=\frac{\partial v_{2}}{\partial x}-\frac{\partial v_{1}}{\partial y}
$$

Also, for a field $\mathbf{v}$ such that its restriction $\left.\mathbf{v}\right|_{T}$ to each $T \in \mathcal{T}_{h}$ belongs to $H^{1}(T)^{2}$ we will denote with $\operatorname{rot}_{h} \mathbf{v}$ the function such that its restriction to $T$ is given by $\operatorname{rot}\left(\left.\mathbf{v}\right|_{T}\right)$.

For an element $T$, let $E_{T}$ be the set of edges of $T$ and $t$ be the unit tangent on $\ell$ oriented clockwise. For an interior side $\ell, \llbracket \mathbf{u}_{h} \cdot t \rrbracket_{\ell}$ denotes the jump of the tangential component of $\mathbf{u}_{h}$, namely, if $T_{1}$ and $T_{2}$ are the 
triangles sharing $\ell$, and $t_{1}$ and $t_{2}$ the corresponding unit tangent vectors on $\ell$ then

$$
\llbracket \mathbf{u}_{h} \cdot t \rrbracket_{\ell}=\left.\mathbf{u}_{h}\right|_{T_{1}} \cdot t_{1}-\left.\mathbf{u}_{h}\right|_{T_{2}} \cdot t_{1}=\left.\mathbf{u}_{h}\right|_{T_{1}} \cdot t_{1}+\left.\mathbf{u}_{h}\right|_{T_{2}} \cdot t_{2} .
$$

We define

$$
J_{\ell}=\left\{\begin{array}{c}
\llbracket \mathbf{u}_{h} \cdot t \rrbracket_{\ell} \text { if } \ell \not \subset \partial \Omega \\
2 \mathbf{u}_{h} \cdot t \text { if } \ell \subset \partial \Omega
\end{array}\right.
$$

We now introduce the estimator for the vector variable and prove the efficiency and reliability of this estimator.

The local error estimator is defined by

$$
\eta_{\text {vect }, T}^{2}=|T|\left\|\operatorname{rot}_{h} \mathbf{u}_{h}\right\|_{L^{2}(T)}^{2}+\sum_{\ell \in E_{T}}|\ell|\left\|J_{\ell}\right\|_{L^{2}(\ell)}^{2}
$$

and the global one by,

$$
\eta_{v e c t}^{2}=\sum_{T \in \mathcal{T}_{h}} \eta_{v e c t, T}^{2}
$$

The key point to prove the reliability of the estimator is to decompose the error by using a generalized Helmholtz decomposition given in the next lemma.

Lemma 4.1 If the domain $\Omega$ is simply connected and $\mathbf{v} \in L^{2}(\Omega)^{2}$, there exist $\psi \in H_{0}^{1}(\Omega)$ and $\phi \in H^{1}(\Omega)$ such that

$$
\mathbf{v}=a \nabla \psi+\operatorname{curl} \phi
$$

and

$$
\|\nabla \phi\|_{L^{2}(\Omega)}+\|\nabla \psi\|_{L^{2}(\Omega)} \leq C\|\mathbf{v}\|_{L^{2}(\Omega)}
$$

with a constant $C$ depending only on a.

Proof. To obtain this decomposition we solve the problem

$$
\operatorname{div}(a \nabla \psi)=\operatorname{div} \mathbf{v}
$$

with $\psi \in H_{0}^{1}(\Omega)$, namely, $\psi$ satisfies

$$
\int_{\Omega} a \nabla \psi \cdot \nabla \xi=\int_{\Omega} \mathbf{v} \cdot \nabla \xi \quad \forall \xi \in H_{0}^{1}(\Omega) .
$$


In particular, choosing $\xi=\psi$ we obtain

$$
\|\nabla \psi\|_{L^{2}(\Omega)} \leq C\|\mathbf{v}\|_{L^{2}(\Omega)} .
$$

Now, since

$$
\operatorname{div}(\mathbf{v}-a \nabla \psi)=0,
$$

and the domain is simply connected, there exists $\phi \in H^{1}(\Omega)$ such that (4.3) holds.

Moreover, observe that (4.4) follows easily from (4.5) and (4.3).

Theorem 4.2 If $\Omega$ is simply connected and the restriction of a to any $T \in$ $\mathcal{T}_{h}$ is constant, there exists a constant $C_{1}$ such that

$$
\left\|\mathbf{u}-\mathbf{u}_{h}\right\|_{L^{2}(\Omega)} \leq C_{1}\left\{\eta_{\text {vect }}+h\left\|f-P_{h} f\right\|_{L^{2}(\Omega)}\right\} .
$$

Proof. For $\phi \in H^{1}(\Omega)$ we have

$$
\int_{\Omega} \mu \mathbf{u} \cdot \operatorname{curl} \phi d x=\int_{\Omega} \nabla p \cdot \operatorname{curl} \phi d x=0 .
$$

Analogously, for $\phi_{h} \in \mathcal{P}_{k+1}^{c}, \operatorname{curl} \phi_{h} \in \mathcal{R} \mathcal{T}_{k}$ and therefore, using the first equation in (3.27),

$$
\int_{\Omega} \mu \mathbf{u}_{h} \cdot \operatorname{curl} \phi_{h} d x=0 .
$$

Then,

$$
\begin{aligned}
& \int_{\Omega} \mu\left(\mathbf{u}-\mathbf{u}_{h}\right) \cdot \operatorname{curl} \phi d x=-\int_{\Omega} \mu \mathbf{u}_{h} \cdot \operatorname{curl}\left(\phi-\phi_{h}\right) d x \\
= & -\sum_{T}\left\{\int_{T} \operatorname{rot}_{h}\left(\mu \mathbf{u}_{h}\right)\left(\phi-\phi_{h}\right) d x+\int_{\partial T} \mu \mathbf{u}_{h} \cdot t\left(\phi-\phi_{h}\right) d s\right\} \\
= & -\sum_{T}\left\{\int_{T} \operatorname{rot}_{h}\left(\mu \mathbf{u}_{h}\right)\left(\phi-\phi_{h}\right) d x+\frac{1}{2} \sum_{\ell \in E_{T}} \int_{\ell} J_{\ell}\left(\phi-\phi_{h}\right) d s\right\}
\end{aligned}
$$

Then, if $\phi_{h} \in \mathcal{P}_{k+1}^{c}$ is an approximation of $\phi$ satisfying (4.1) and (4.2), applying the Schwarz inequality we obtain

$$
\int_{\Omega} \mu\left(\mathbf{u}-\mathbf{u}_{h}\right) \cdot \operatorname{curl} \phi d x \leq C \eta_{v e c t}|\phi|_{1, \Omega} .
$$

On the other hand, if $\psi \in H_{0}^{1}(\Omega)$ we have 


$$
\begin{gathered}
\int_{\Omega} \mu\left(\mathbf{u}-\mathbf{u}_{h}\right) \cdot a \nabla \psi d x=\int_{\Omega}\left(\mathbf{u}-\mathbf{u}_{h}\right) \cdot \nabla \psi d x \\
=\int_{\Omega} \operatorname{div}\left(\mathbf{u}-\mathbf{u}_{h}\right) \psi d x=\int_{\Omega}\left(f-P_{h} f\right) \psi d x=\int_{\Omega}\left(f-P_{h} f\right)\left(\psi-P_{h} \psi\right) d x
\end{gathered}
$$

and, therefore, using that

$$
\left\|\psi-P_{h} \psi\right\|_{L^{2}(\Omega)} \leq C h\|\nabla \psi\|_{L^{2}(\Omega)},
$$

which follows immediately from Corollary 2.3, we obtain

$$
\int_{\Omega}\left(\mathbf{u}-\mathbf{u}_{h}\right) \cdot \nabla \psi d x \leq C h\left\|f-P_{h} f\right\|_{L^{2}(\Omega)}\|\nabla \psi\|_{L^{2}(\Omega)} .
$$

Using now Lemma 4.3 for $\mathbf{v}=\mathbf{u}-\mathbf{u}_{h}$ we have

$$
\mathbf{u}-\mathbf{u}_{h}=a \nabla \psi+\operatorname{curl} \phi
$$

with $\psi \in H_{0}^{1}(\Omega)$ and $\phi \in H^{1}(\Omega)$ such that

$$
\|\nabla \phi\|_{L^{2}(\Omega)}+\|\nabla \psi\|_{L^{2}(\Omega)} \leq C\left\|\mathbf{u}-\mathbf{u}_{h}\right\|_{L^{2}(\Omega)} .
$$

Then,

$$
\left\|\mathbf{u}-\mathbf{u}_{h}\right\|_{L^{2}(\Omega)}^{2} \leq C\left\{\int_{\Omega} \mu\left(\mathbf{u}-\mathbf{u}_{h}\right) \cdot \operatorname{curl} \phi d x+\int_{\Omega}\left(\mathbf{u}-\mathbf{u}_{h}\right) \cdot \nabla \psi d x\right\}
$$

and therefore (4.6) follows immediately from (4.7), (4.8) and (4.9).

To prove the efficiency we will use a well-known argument of Verfürth $[50,52]$. In our case this argument will make use of the following lemma.

Lemma 4.3 Given a triangle $T$ and functions $q_{T} \in L^{2}(T)$ and, for each side $\ell$ of $T, p_{\ell} \in L^{2}(\ell)$, there exists $\phi \in \mathcal{P}_{k+3}(T)$ such that

$$
\left\{\begin{array}{crl}
\int_{T} \phi r d x=\int_{T} q_{T} r d x & \forall r \in \mathcal{P}_{k}(T) \\
\int_{\ell} \phi s d x=\int_{\ell} p_{\ell} s d x & \forall s \in \mathcal{P}_{k+1}(\ell) \forall \ell \in E_{T} \\
\phi=0 & \text { at the vertices of } T
\end{array}\right.
$$

Moreover,

$$
\|\nabla \phi\|_{L^{2}(T)} \leq C\left\{|T|^{-\frac{1}{2}}\left\|q_{T}\right\|_{L^{2}(T)}+\sum_{\ell \in E_{T}}|\ell|^{-\frac{1}{2}}\left\|p_{\ell}\right\|_{L^{2}(\ell)}\right\}
$$


Proof. The number of conditions is

$$
\operatorname{dim} \mathcal{P}_{k}(T)+3 \operatorname{dim} \mathcal{P}_{k+1}(\ell)=\frac{(k+2)(k+1)}{2}+3(k+2)=\frac{(k+2)(k+7)}{2}
$$

while the dimension of the subspace of $\mathcal{P}_{k+3}$ of polynomials vanishing at the vertices of $T$ is

$$
\operatorname{dim} \mathcal{P}_{k+3}(T)-3=\frac{(k+4)(k+5)}{2}-3==\frac{(k+2)(k+7)}{2}
$$

Therefore, (4.10) is a square system and so it is enough to show the uniqueness. So, assume that

$$
\left\{\begin{array}{lr}
\int_{T} \phi r d x=0 & \forall r \in \mathcal{P}_{k}(T) \\
\int_{\ell} \phi s d x=0 & \forall s \in \mathcal{P}_{k+1}(\ell) \forall \ell \in E_{T} \\
\phi=0 & \text { at the vertices of } T .
\end{array}\right.
$$

Since $\phi$ vanishes at the vertices of $\ell$, it follows from the second condition in (4.12) that $\phi=0$ on the sides of $T$. Then,

$$
\phi=\lambda_{1} \lambda_{2} \lambda_{3} r \quad \text { with } r \in \mathcal{P}_{k}
$$

and, therefore, it follows from the first condition in (4.12) that $\phi=0$.

We will call $D_{T}$ the union of $T$ with the triangles sharing a side with it.

Theorem 4.4 If the restriction of a to any $T \in \mathcal{T}_{h}$ is constant, there exists a constant $C_{2}$ such that, for any $T \in \mathcal{T}_{h}$,

$$
\eta_{v e c t, T} \leq C_{2}\left\|\mathbf{u}-\mathbf{u}_{h}\right\|_{L^{2}\left(D_{T}\right)}
$$

Proof. We apply Lemma 4.3 on $T$ and its neighbors $T_{i}, i=1,2,3$ (we assume that $T$ does not have a side on $\partial \Omega$, trivial modifications are needed if this is not the case). In this way we can construct $\phi \in H_{0}^{1}\left(D_{T}\right)$ vanishing at the vertices of $T$ and $T_{i}, i=1,2,3$ and such that

$$
\begin{array}{cc}
\int_{T} \phi r d x=-\int_{T}|T| \operatorname{rot}_{h}\left(\mu \mathbf{u}_{h}\right) r d x & \forall r \in \mathcal{P}_{k}(T) \\
\int_{\ell} \phi s d x=-\int_{\ell}|\ell| J_{\ell} s d x & \forall s \in \mathcal{P}_{k+1}(\ell) \quad, \forall \ell \in E_{T},
\end{array}
$$




$$
\int_{T_{i}} \phi r d x=0 \quad \forall r \in \mathcal{P}_{k}\left(T_{i}\right)
$$

and

$$
\int_{\ell} \phi s d x=0 \quad \forall s \in \mathcal{P}_{k+1}(\ell) \quad \text { on the other two sides of } T_{i} .
$$

Since $\phi$ vanishes at the boundary of $D_{T}$ we can extend it by zero to obtain a function $\phi \in H_{0}^{1}(\Omega)$. Then,

$$
\int_{\Omega} \mu\left(\mathbf{u}-\mathbf{u}_{h}\right) \cdot \operatorname{curl} \phi d x=-\sum_{T}\left\{\int_{T} \operatorname{rot}_{h}\left(\mu \mathbf{u}_{h}\right) \phi d x+\frac{1}{2} \sum_{\ell \in E_{T}} \int_{\ell} J_{\ell} \phi d s\right\} .
$$

But,

$$
\left.\operatorname{rot}_{h}\left(\mu \mathbf{u}_{h}\right)\right|_{T} \in \mathcal{P}_{k}(T) \text { and } J_{\ell} \in \mathcal{P}_{k+1}(\ell),
$$

therefore, we can take $r=\operatorname{rot}\left(\mu \mathbf{u}_{h}\right)$ and, for each $\ell \in E_{T}, s=J_{\ell}$ in (4.14) and (4.15) respectively to obtain

$$
\int_{T} \operatorname{rot}_{h}\left(\mu \mathbf{u}_{h}\right) \phi d x=-|T|\left\|\operatorname{rot}_{h} \mathbf{u}_{h}\right\|_{L^{2}(T)}^{2}
$$

and

$$
\sum_{\ell \in E_{T}} \int_{\ell} J_{\ell} \phi d s=-\sum_{\ell \in E_{T}}|\ell|\left\|J_{\ell}\right\|_{L^{2}(\ell)}^{2}
$$

Analogously, using now (4.15), (4.16), (4.17), we obtain

$$
\int_{T_{i}} \operatorname{rot}_{h}\left(\mu \mathbf{u}_{h}\right) \phi d x=0 \quad, \quad i=1,2,3
$$

and

$$
\sum_{\tilde{\ell} \in E_{T_{i}}} \int_{\tilde{\ell}} J_{\tilde{\ell}} \phi d s=-|\ell|\left\|J_{\ell}\right\|_{L^{2}(\ell)}^{2} \quad, \quad i=1,2,3
$$

where $\ell=T \cap T_{i}$.

Therefore, recalling that $\phi$ vanishes outside $D_{T}$, it follows from (4.18) that

$$
\eta_{v e c t, T}^{2}=\int_{D_{T}} \mu\left(\mathbf{u}-\mathbf{u}_{h}\right) \cdot \operatorname{curl} \phi d x
$$

and so,

$$
\eta_{\text {vect }, T}^{2} \leq C\left\|\mathbf{u}-\mathbf{u}_{h}\right\|_{L^{2}(\Omega)}\|\nabla \phi\|_{L^{2}\left(D_{T}\right)} .
$$


But, using (4.11) we have

$$
\|\nabla \phi\|_{L^{2}\left(D_{T}\right)} \leq C\left\{|T|^{\frac{1}{2}}\left\|\operatorname{rot}_{h}\left(\mu \mathbf{u}_{h}\right)\right\|_{L^{2}(T)}+\sum_{\ell \in E_{T}}|\ell|^{\frac{1}{2}}\left\|J_{\ell}\right\|_{L^{2}(\ell)}\right\} \leq C \eta_{\text {vect }, T}
$$

and therefore (4.13) holds.

To estimate the error in the scalar variable $p$ we introduce the local estimator

$$
\eta_{e s c, T}^{2}=|T|\left\|\nabla_{h} p_{h}+\mu \mathbf{u}_{h}\right\|_{L^{2}(T)}^{2}+\sum_{\ell \in E_{T}}|\ell|\left\|\llbracket p_{h} \rrbracket_{\ell}\right\|_{L^{2}(\ell)}^{2}
$$

where $\llbracket p_{h} \rrbracket_{\ell}$ denotes the jump of $p_{h}$ across the side $\ell$ if $\ell$ is an interior side or $\llbracket p_{h} \rrbracket_{\ell}=2 p_{h}$ if $\ell \subset \partial \Omega$ and, for a function $q$ such that its restriction to each $T \in \mathcal{T}_{h}$ belongs to $H^{1}(T)$ we denote with $\nabla_{h} q$ the function such that its restriction to $T$ is given by $\nabla\left(\left.q\right|_{T}\right)$

Then, the global estimator is defined as usual by

$$
\eta_{e s c}^{2}=\sum_{T \in \mathcal{T}_{h}} \eta_{e s c, T}^{2}
$$

The next lemma shows that the error in the scalar variable is bounded by $\eta_{\text {esc }}$ plus the error in the vector variable.

Apart from (3.18) we will use the following error estimate which can be obtained in a similar way.

If $\ell$ is a side of an element $T$ we have

$$
\left\|\left(\mathbf{v}-\Pi_{T} \mathbf{v}\right) \cdot \mathbf{n}\right\|_{L^{2}(\ell)} \leq C|\ell|^{\frac{1}{2}}\|\nabla \mathbf{v}\|_{L^{2}(T)} .
$$

Lemma 4.5 There exists a constant $C$ such that

$$
\left\|p-p_{h}\right\|_{L^{2}(\Omega)} \leq C\left\{\eta_{e s c}+\left\|\mathbf{u}-\mathbf{u}_{h}\right\|_{L^{2}(\Omega)}\right\} .
$$

Proof. By Lemma 2.4 we know that there exists $\mathbf{v} \in H^{1}(\Omega)^{2}$ such that

$$
\operatorname{div} \mathbf{v}=p-p_{h}
$$

and

$$
\|\mathbf{v}\|_{H^{1}(\Omega)} \leq C\left\|p-p_{h}\right\|_{L^{2}(\Omega)}
$$

with a constant $C$ depending only on the domain. 
Then,

$$
\begin{aligned}
& \left\|p-p_{h}\right\|_{L^{2}(\Omega)}^{2}=\int_{\Omega}\left(p-p_{h}\right) \operatorname{div} \mathbf{v} d x=\int_{\Omega}\left(p-p_{h}\right) \operatorname{div}\left(\mathbf{v}-\Pi_{h} \mathbf{v}\right) d x+\int_{\Omega}\left(p-p_{h}\right) \operatorname{div} \Pi_{h} \mathbf{v} d x \\
& =\int_{\Omega}\left(p-p_{h}\right) \operatorname{div}\left(\mathbf{v}-\Pi_{h} \mathbf{v}\right) d x-\int_{\Omega} \mu\left(\mathbf{u}-\mathbf{u}_{h}\right) \cdot\left(\mathbf{v}-\Pi_{h} \mathbf{v}\right) d x+\int_{\Omega} \mu\left(\mathbf{u}-\mathbf{u}_{h}\right) \cdot \mathbf{v} d x .
\end{aligned}
$$

But using that

$$
\int_{\Omega} p \operatorname{div}\left(\mathbf{v}-\Pi_{h} \mathbf{v}\right) d x-\int_{\Omega} \mu \mathbf{u} \cdot\left(\mathbf{v}-\Pi_{h} \mathbf{v}\right) d x=0
$$

and integrating by parts on each element we have

$$
\begin{gathered}
\int_{\Omega}\left(p-p_{h}\right) \operatorname{div}\left(\mathbf{v}-\Pi_{h} \mathbf{v}\right) d x-\int_{\Omega} \mu\left(\mathbf{u}-\mathbf{u}_{h}\right) \cdot\left(\mathbf{v}-\Pi_{h} \mathbf{v}\right) d x \\
=\sum_{T \in \mathcal{T}_{h}}\left\{\int_{T} \nabla_{h} p_{h} \cdot\left(\mathbf{v}-\Pi_{h} \mathbf{v}\right) d x-\int_{\partial T} p_{h}\left(\mathbf{v}-\Pi_{h} \mathbf{v}\right) \cdot \mathbf{n} d s+\int_{T} \mu \mathbf{u}_{h} \cdot\left(\mathbf{v}-\Pi_{h} \mathbf{v}\right) d x\right\} \\
=\sum_{T \in \mathcal{T}_{h}}\left\{\int_{T}\left(\nabla_{h} p_{h}+\mu \mathbf{u}_{h}\right) \cdot\left(\mathbf{v}-\Pi_{h} \mathbf{v}\right) d x-\frac{1}{2} \sum_{\ell \in E_{T}} \int_{\ell} \llbracket p_{h} \rrbracket\left(\mathbf{v}-\Pi_{h} \mathbf{v}\right) \cdot \mathbf{n} d s\right\} .
\end{gathered}
$$

Therefore, the Lemma follows from this equality and (4.22) using the Schwarz inequality and the error estimates (3.18) and (4.19).

Using now the results for the vector variable we obtain the following a posteriori error estimate for the scalar variable.

Theorem 4.6 If $\Omega$ is simply connected and the restriction of a to any $T \in$ $\mathcal{T}_{h}$ is constant, there exists a constant $C_{3}$ such that

$$
\left\|p-p_{h}\right\|_{L^{2}(\Omega)} \leq C_{3}\left\{\eta_{\text {esc }}+\eta_{\text {vect }}+h\left\|f-P_{h} f\right\|_{L^{2}(\Omega)}\right\}
$$

Proof. This result follows immediately from Theorem 4.6 and Lemma 4.5.

To prove the efficiency of $\eta_{\text {esc }}$ we first prove that the jumps involved in the definition of the estimator can be bounded by the error plus the other part of the estimator.

Lemma 4.7 There exists a constant $C$ such that

$|\ell|^{\frac{1}{2}}\left\|\llbracket p_{h} \rrbracket\right\|_{L^{2}(\ell)} \leq C\left\{\left\|p-p_{h}\right\|_{L^{2}\left(D_{\ell}\right)}+|\ell|\left\|\mathbf{u}-\mathbf{u}_{h}\right\|_{L^{2}\left(D_{\ell}\right)}+|\ell|\left\|\nabla_{h} p_{h}+\mu \mathbf{u}_{h}\right\|_{L^{2}\left(D_{\ell}\right)}\right\}$ where $D_{\ell}$ is the union of the triangles sharing $\ell$. 
Proof. If $\ell \in E_{T}$, it follows from the regularity assumption on the meshes that $|\ell| \sim h_{T} \sim|T|^{\frac{1}{2}}$. Now, since $p$ is continuous we have

$$
\left\|\llbracket p_{h} \rrbracket\right\|_{L^{2}(\ell)}=\left\|\llbracket p_{h}-p \rrbracket\right\|_{L^{2}(\ell)}
$$

and so, applying the trace inequality (2.1), we obtain

$$
\begin{gathered}
|\ell|^{\frac{1}{2}}\left\|\llbracket p_{h} \rrbracket\right\|_{L^{2}(\ell)} \leq C\left\{\left\|p_{h}-p\right\|_{L^{2}\left(D_{\ell}\right)}+|\ell|\left\|\nabla_{h}\left(p_{h}-p\right)\right\|_{L^{2}\left(D_{\ell}\right)}\right\} \\
\leq C\left\{\left\|p_{h}-p\right\|_{L^{2}\left(D_{\ell}\right)}+|\ell|\left\|\nabla_{h} p_{h}+\mu \mathbf{u}\right\|_{L^{2}\left(D_{\ell}\right)}\right\} \\
\leq C\left\{\left\|p_{h}-p\right\|_{L^{2}\left(D_{\ell}\right)}+|\ell|\left\|\nabla_{h} p_{h}+\mu \mathbf{u}_{h}\right\|_{L^{2}\left(D_{\ell}\right)}+|\ell|\left\|\mu\left(\mathbf{u}-\mathbf{u}_{h}\right)\right\|_{L^{2}\left(D_{\ell}\right)}\right\}
\end{gathered}
$$

concluding the proof because $\mu$ is bounded.

Now, in order to bound $\left\|\nabla_{h} p_{h}+\mu \mathbf{u}\right\|_{L^{2}(T)}$ by the error we will use again the argument of Verfürth.

Lemma 4.8 There exists a constant $C$ such that

$$
|T|^{\frac{1}{2}}\left\|\nabla_{h} p_{h}+\mu \mathbf{u}_{h}\right\|_{L^{2}(T)} \leq C\left\{|T|^{\frac{1}{2}}\left\|\mathbf{u}-\mathbf{u}_{h}\right\|_{L^{2}(T)}+\left\|p-p_{h}\right\|_{L^{2}(T)}\right\}
$$

Proof. Using again that

$$
\int_{\Omega} \mu \mathbf{u} \cdot \mathbf{v} d x-\int_{\Omega} p \operatorname{div} \mathbf{v} d x=0
$$

we have, for any $\mathbf{v} \in H_{0}^{1}(T)$,

$$
\begin{aligned}
\int_{T} \mu\left(\mathbf{u}-\mathbf{u}_{h}\right) \cdot \mathbf{v} d x-\int_{T}\left(p-p_{h}\right) \operatorname{div} \mathbf{v} d x & =-\int_{T} \mu \mathbf{u}_{h} \cdot \mathbf{v} d x+\int_{T} p_{h} \operatorname{div} \mathbf{v} d x \\
=-\int_{T} \mu \mathbf{u}_{h} \cdot \mathbf{v} d x-\int_{T} \nabla_{h} p_{h} \cdot \mathbf{v} d x & =-\int_{T}\left(\nabla_{h} p_{h}+\mu \mathbf{u}_{h}\right) \cdot \mathbf{v} d x
\end{aligned}
$$

Choosing now $\mathbf{v}=-b_{T}\left(\nabla_{h} p_{h}+\mu \mathbf{u}_{h}\right)$, with $b_{T} \in \mathcal{P}_{3}(T)$ vanishing at the boundary and equal to one at the barycenter of $T$, we obtain

$$
\int_{T} \mu\left(\mathbf{u}-\mathbf{u}_{h}\right) \cdot \mathbf{v} d x-\int_{T}\left(p-p_{h}\right) \operatorname{div} \mathbf{v} d x=\int_{T}\left|\nabla_{h} p_{h}+\mu \mathbf{u}_{h}\right|^{2} b_{T} d x .
$$

But, since $\nabla_{h} p_{h}+\mu \mathbf{u}_{h} \in \mathcal{P}_{k+1}(T)$, a standard argument (equivalence of norms in a reference element and an affine change of variables) gives

$$
\int_{T}\left|\nabla_{h} p_{h}+\mu \mathbf{u}_{h}\right|^{2} d x \leq C \int_{T}\left|\nabla_{h} p_{h}+\mu \mathbf{u}_{h}\right|^{2} b_{T} d x
$$


which together with (4.25) and the Schwarz inequality yields

$$
\left\|\nabla_{h} p_{h}+\mu \mathbf{u}_{h}\right\|_{L^{2}(T)}^{2} \leq C\left\{\left\|\mathbf{u}-\mathbf{u}_{h}\right\|_{0, T}\|\mathbf{v}\|_{L^{2}(T)}+\left\|p-p_{h}\right\|_{L^{2}(T)}\|\nabla \mathbf{v}\|_{L^{2}(T)}\right\}
$$

but, since $b_{T}$ is bounded by a constant independent of $T$ we know that

$$
\|\mathbf{v}\|_{L^{2}(T)} \leq C\left\|\nabla_{h} p_{h}+\mu \mathbf{u}_{h}\right\|_{L^{2}(T)}
$$

and, by a standard inverse inequality,

$$
\|\nabla \mathbf{v}\|_{L^{2}(T)} \leq C|T|^{-\frac{1}{2}}\left\|\nabla_{h} p_{h}+\mu \mathbf{u}_{h}\right\|_{L^{2}(T)}
$$

and, therefore, (4.24) follows from (4.26).

Collecting the lemmas we can prove the efficiency of the estimator $\eta_{\text {esc }}$.

Theorem 4.9 If the restriction of a to any $T \in \mathcal{T}_{h}$ is constant, there exists a constant $C_{4}$ such that, for any $T \in \mathcal{T}_{h}$,

$$
\eta_{e s c, T} \leq C_{4}\left\{|T|^{\frac{1}{2}}\left\|\mathbf{u}-\mathbf{u}_{h}\right\|_{L^{2}\left(D_{T}\right)}+\left\|p-p_{h}\right\|_{L^{2}\left(D_{T}\right)}\right\}
$$

Proof. This result is an immediate consequence of Lemmas 4.7 and 4.8 .

Putting together the results for both estimators we have the following a posteriori error estimate for the mixed finite element approximation.

We define

$$
\eta_{T}^{2}=\eta_{v e c t, T}^{2}+\eta_{e s c, T}^{2} \quad \text { and } \quad \eta^{2}=\sum_{T \in \mathcal{T}_{h}} \eta_{T}^{2}
$$

Theorem 4.10 If $\Omega$ is simply connected and the restriction of a to any $T \in \mathcal{T}_{h}$ is constant, there exist constants $C_{5}$ and $C_{6}$ such that

$$
\eta_{T} \leq C_{5}\left\{\left\|\mathbf{u}-\mathbf{u}_{h}\right\|_{L^{2}\left(D_{T}\right)}+\left\|p-p_{h}\right\|_{L^{2}\left(D_{T}\right)}\right\}
$$

and

$$
\left\|\mathbf{u}-\mathbf{u}_{h}\right\|_{L^{2}(\Omega)}+\left\|p-p_{h}\right\|_{L^{2}(\Omega)} \leq C_{6}\left\{\eta+h\left\|f-P_{h} f\right\|_{L^{2}(\Omega)}\right\} .
$$

Proof. This result is an immediate consequence of Theorems 4.2, 4.4, 4.6 and 4.9 . 


\section{The general abstract setting}

The problem considered in the previous sections is a particular case of a general class of problems that we are going to analize in this section. The theory presented here was first developed by Brezzi [13]. Some of the ideas were also introduced for particular problems by Babuska [9] and by Crouzeix and Raviart [22] We also refer the reader to $[32,31]$ and to the books $[17$, $45,37]$.

Let $V$ and $Q$ be two Hilbert spaces and suppose that $a($,$) and b($,$) are$ continuous bilinear forms on $V \times V$ and $V \times Q$ respectively, i.e.,

$$
|a(u, v)| \leq\|a\|\|u\|_{V}\|v\|_{V} \quad \forall u \in V, \forall v \in V
$$

and

$$
|b(v, q)| \leq\|b\|\|v\|_{V}\|q\|_{Q} \quad \forall v \in V, \forall q \in Q
$$

Consider the problem: given $f \in V^{\prime}$ and $g \in Q^{\prime}$ find $(u, p) \in V \times Q$ solution of

$$
\left\{\begin{aligned}
a(u, v)+b(v, p) & =\langle f, v\rangle & & \forall v \in V \\
b(u, q) & =\langle g, q\rangle & & \forall q \in Q
\end{aligned}\right.
$$

where $\langle.,$.$\rangle denotes the duality product between a space and its dual one.$

For example, the mixed formulation of second order elliptic problems considered in the previous sections can be written in this way with

$$
V=H(\operatorname{div}, \Omega) \quad, \quad Q=L^{2}(\Omega)
$$

and

$$
a(\mathbf{u}, \mathbf{v})=\int_{\Omega} \mu \mathbf{u} \cdot \mathbf{v} d x \quad, \quad b(\mathbf{v}, p)=\int_{\Omega} p \operatorname{div} \mathbf{v} d x .
$$

The general problem (5.1) can be written in the standard way

$$
c((u, p),(v, q))=\langle f, v\rangle+\langle g, q\rangle \quad \forall(v, q) \in V \times Q
$$

where $c$ is the continuous bilinear form on $V \times Q$ defined by

$$
c((u, p),(v, q))=a(u, v)+b(v, p)+b(u, q) .
$$

However, the bilinear form is not coercive and therefore the usual finite element error analysis can not be applied. 
We will give sufficient conditions (indeed, they are also necessary although we are not going to prove it here, we refer to $[17,37])$ on the forms $a$ and $b$ for the existence and uniqueness of a solution of problem (5.1). Below, we will also show that their discrete version ensures the stability and optimal order error estimates for the Galerkin approximations. These results were obtained by Brezzi [13] (see also [17] were more general results are proved).

Introducing the continuous operators $A: V \rightarrow V^{\prime}, B: V \rightarrow Q^{\prime}$ and its adjoint $B^{*}: Q \rightarrow V^{\prime}$ defined by,

$$
\langle A u, v\rangle_{V^{\prime} \times V}=a(u, v)
$$

and

$$
\langle B v, q\rangle_{Q^{\prime} \times Q}=b(v, q)=\left\langle v, B^{*} q\right\rangle_{V \times V^{\prime}}
$$

problem (5.1) can also be written as

$$
\left\{\begin{aligned}
A u+B^{*} p & =f & & \text { in } V^{\prime} \\
B u & =g & & \text { in } Q^{\prime}
\end{aligned}\right.
$$

Let us introduce $W=\operatorname{Ker} B \subset V$ and, for $g \in Q^{\prime}$,

$$
W(g)=\{v \in V: B v=g\}
$$

Now, if $(u, p) \in V \times Q$ is a solution of (5.1) then, it is easy to see that $u \in W(g)$ is a solution of the problem

$$
a(u, v)=\langle f, v\rangle \quad \forall v \in W .
$$

We will find conditions under which both problems (5.1) and (5.4) are equivalent, in the sense that for a solution $u \in W(g)$ of (5.4) there exists a unique $p \in Q$ such that $(u, p)$ is a solution of (5.1).

In what follows we will use the following well-known result of functional analysis. Given a Hilbert space $V$ and $S \subset V$ we define $S^{0} \subset V^{\prime}$ by

$$
S^{0}=\left\{L \in V^{\prime}:\langle L, v\rangle=0, \quad \forall v \in S\right\}
$$

Theorem 5.1 Let $V_{1}$ and $V_{2}$ be Hilbert spaces and $A: V_{1} \rightarrow V_{2}^{\prime}$ be a continuous linear operator. Then,

$$
(\operatorname{Ker} A)^{0}=\overline{\operatorname{Im} A^{*}}
$$

and

$$
\left(\operatorname{Ker} A^{*}\right)^{0}=\overline{\operatorname{ImA}}
$$


Proof. It is easy to see that $\operatorname{Im} A^{*} \subset(\operatorname{Ker} A)^{0}$ and that $(\operatorname{Ker} A)^{0}$ is a closed subspace of $V_{1}^{\prime}$. Therefore

$$
\overline{\operatorname{Im} A^{*}} \subset(\operatorname{Ker} A)^{0} .
$$

Suppose now that there exists $L_{0} \in V_{1}^{\prime}$ such that $L_{0} \in(\operatorname{Ker} A)^{0} \backslash \overline{\operatorname{Im} A^{*} \text {. }}$ Then, by the Hahn-Banach theorem there exists a linear continuous functional defined on $V_{1}^{\prime}$ which vanishes on $\overline{\operatorname{Im} A^{*}}$ and is different from zero on $L_{0}$. In other words, using the standard identification between $V_{1}^{\prime \prime}$ and $V_{1}$, there exists $v_{0} \in V_{1}$ such that

$$
\left\langle L_{0}, v_{0}\right\rangle \neq 0 \quad \text { and } \quad\left\langle L, v_{0}\right\rangle=0 \quad \forall L \in \overline{\operatorname{Im} A^{*}} .
$$

In particular, for all $v \in V_{2}$

$$
\left\langle A v_{0}, v\right\rangle=\left\langle v_{0}, A^{*} v\right\rangle=0
$$

and so $v_{0} \in \operatorname{Ker} A$ which, since $L_{0} \in(\operatorname{Ker} A)^{0}$, contradicts $\left\langle L_{0}, v_{0}\right\rangle \neq$ 0. Therefore, $(\operatorname{Ker} A)^{0} \subset \overline{\operatorname{Im} A^{*}}$ and so (5.5) holds. Finally, (5.6) is an immediate consequence of (5.5) because $\left(A^{*}\right)^{*}=A$.

Lemma 5.2 The following properties are equivalent:

a) There exists $\beta>0$ such that

$$
\sup _{v \in V} \frac{b(v, q)}{\|v\|_{V}} \geq \beta\|q\|_{Q} \quad \forall q \in Q
$$

b) $B^{*}$ is an isomorphism from $Q$ onto $W^{0}$ and,

$$
\left\|B^{*} q\right\|_{V^{\prime}} \geq \beta\|q\|_{Q} \quad \forall q \in Q
$$

c) $B$ is an isomorphism from $W^{\perp}$ onto $Q^{\prime}$ and,

$$
\|B v\|_{Q^{\prime}} \geq \beta\|v\|_{V} \quad \forall v \in W^{\perp}
$$

Proof. Assume that $a$ ) holds then, (5.8) is satisfied and so $B^{*}$ is injective. Moreover $\operatorname{Im} B^{*}$ is a closed subspace of $V^{\prime}$, indeed, suppose that $B^{*} q_{n} \rightarrow w$ then, it follows from (5.8) that

$$
\left\|B^{*}\left(q_{n}-q_{m}\right)\right\|_{V^{\prime}} \geq \beta\left\|q_{n}-q_{m}\right\|_{Q}
$$


and, therefore, $\left\{q_{n}\right\}$ is a Cauchy sequence and so it converges to some $q \in Q$ and, by continuity of $B^{*}, w=B^{*} q \in \operatorname{Im} B^{*}$. Consequently, using (5.5) we obtain that $\operatorname{Im} B^{*}=W^{0}$ and therefore $b$ ) holds.

Now, we observe that $W^{0}$ can be isometrically identified with $\left(W^{\perp}\right)^{\prime}$. Indeed, denoting with $P^{\perp}: V \rightarrow W^{\perp}$ the orthogonal projection, for any $g \in\left(W^{\perp}\right)^{\prime}$ we define $\tilde{g} \in W^{0}$ by $\tilde{g}=g \circ P^{\perp}$ and it is easy to check that $g \rightarrow \tilde{g}$ is an isometric bijection from $\left(W^{\perp}\right)^{\prime}$ onto $W^{0}$ and then, we can identify these two spaces. Therefore $b$ ) and $c$ ) are equivalent.

Corollary 5.3 If the form b satisfies (5.7) then, problems (5.1) and (5.4) are equivalent, that is, there exists a unique solution of (5.1) if and only if there exists a unique solution of (5.4).

Proof. If $(u, p)$ is a solution of (5.1) we know that $u \in W(g)$ and that it is a solution of (5.4). It rests only to check that for a solution $u \in W(g)$ of (5.4) there exists a unique $p \in Q$ such that $B^{*} p=f-A u$ but, this follows from $b$ ) of the previous lemma since, as it is easy to check, $f-A u \in W^{0}$.

Now we can prove the fundamental existence and uniqueness theorem for problem (5.1).

Lemma 5.4 If there exists $\alpha>0$ such that a satisfies

$$
\begin{aligned}
& \sup _{v \in W} \frac{a(u, v)}{\|v\|_{V}} \geq \alpha\|u\|_{V} \quad \forall u \in W \\
& \sup _{u \in W} \frac{a(u, v)}{\|u\|_{V}} \geq \alpha\|v\|_{V} \quad \forall \mathrm{v} \in \mathrm{W}
\end{aligned}
$$

then, for any $g \in W^{\prime}$ there exists $w \in W$ such that

$$
a(w, v)=\langle g, v\rangle \quad \forall v \in W
$$

and moreover

$$
\|w\|_{W} \leq \frac{1}{\alpha}\|g\|_{W^{\prime}}
$$

Proof. Considering the operators

$$
A: W \rightarrow W^{\prime} \text { and } A^{*}: W \rightarrow W^{\prime}
$$


defined by

$$
\langle A u, v\rangle_{W^{\prime} \times W}=a(u, v) \text { and }\left\langle u, A^{*} v\right\rangle_{W \times W^{\prime}}=a(u, v),
$$

conditions (5.10) and (5.11) can be written as

$$
\|A u\|_{W^{\prime}} \geq \alpha\|u\|_{W} \quad \forall u \in W
$$

and

$$
\left\|A^{*} v\right\|_{W^{\prime}} \geq \alpha\|v\|_{W} \quad \forall v \in W
$$

respectively. Therefore, it follows from (5.11) that

$$
\operatorname{Ker} A^{*}=\{0\} .
$$

Then, from (5.6), we have

$$
\left(\operatorname{Ker} A^{*}\right)^{0}=\overline{\operatorname{ImA}}
$$

and so

$$
\overline{\operatorname{Im} A}=W^{\prime} .
$$

Using now (5.13) and the same argument used in Lemma 5.7 to prove that $\operatorname{Im} B^{*}$ is closed, we can show that $\operatorname{Im} A$ is a closed subspace of $W^{\prime}$ and consequently $\operatorname{Im} A=W^{\prime}$ as we wanted to show. Finally (5.12) follows immediately from (5.13). $\square$

Theorem 5.5 If a satisfies (5.10) and (5.11), and $b$ satisfies (5.7) then, there exists a unique solution $(u, p) \in V \times Q$ of problem (5.1) and moreover,

$$
\|u\|_{V} \leq \frac{1}{\alpha}\|f\|_{V^{\prime}}+\frac{1}{\beta}\left(1+\frac{\|a\|}{\alpha}\right)\|g\|_{Q^{\prime}}
$$

and,

$$
\|p\|_{Q} \leq \frac{1}{\beta}\left(1+\frac{\|a\|}{\alpha}\right)\|f\|_{V^{\prime}}+\frac{\|a\|}{\beta^{2}}\left(1+\frac{\|a\|}{\alpha}\right)\|g\|_{Q^{\prime}}
$$

Proof. First we show that there exists a solution $u \in W(g)$ of problem (5.4). Since (5.7) holds we know from Lemma 5.2 that there exists a unique $u_{0} \in W^{\perp}$ such that $B u_{0}=g$ and

$$
\left\|u_{0}\right\|_{V} \leq \frac{1}{\beta}\|g\|_{Q^{\prime}}
$$


then, the existence of $u \in W(g)$ solution of (5.4) is equivalent to the existence of $w=u-u_{0} \in W$ such that

$$
a(w, v)=\langle f, v\rangle-a\left(u_{0}, v\right) \quad \forall v \in W
$$

but, from Lemma 5.4, it follows that such a $w$ exists and moreover,

$$
\|w\|_{V} \leq \frac{1}{\alpha}\left\{\|f\|_{V^{\prime}}+\|a\|\left\|u_{0}\right\|_{V}\right\} \leq \frac{1}{\alpha}\left\{\|f\|_{V^{\prime}}+\frac{\|a\|}{\beta}\|g\|_{Q^{\prime}}\right\}
$$

where we have used (5.17).

Therefore, $u=w+u_{0}$ is a solution of (5.4) and satisfies (5.15).

Now, from Corollary 5.3 it follows that there exists a unique $p \in Q$ such that $(u, p)$ is a solution of (5.1). On the other hand, from Lemma 5.2 it follows that (5.8) holds and using it, it is easy to check that

$$
\|p\|_{Q} \leq \frac{1}{\beta}\left\{\|f\|_{V^{\prime}}+\|a\|\|u\|_{V}\right\}
$$

which combined with (5.15) yields (5.16). Finally, the uniqueness of solution follows from (5.15) and (5.16).

Assume now that we have two families of subspaces $V_{h} \subset V$ and $Q_{h} \subset Q$. We can define the Galerkin approximation $\left(u_{h}, p_{h}\right) \in V_{h} \times Q_{h}$ to the solution $(u, p) \in V \times Q$ of problem (5.1), i.e., $\left(u_{h}, p_{h}\right)$ satisfies,

$$
\left\{\begin{aligned}
a\left(u_{h}, v\right)+b\left(v, p_{h}\right) & =\langle f, v\rangle & & \forall v \in V_{h} \\
b\left(u_{h}, q\right) & =\langle g, q\rangle & & \forall q \in Q_{h}
\end{aligned}\right.
$$

For the error analysis it is convenient to introduce the associated operator $B_{h}: V_{h} \rightarrow Q_{h}^{\prime}$ defined by

$$
\left\langle B_{h} v, q\right\rangle_{Q_{h}^{\prime} \times Q_{h}}=b(v, q)
$$

and the subsets of $V_{h}, W_{h}=\operatorname{Ker} B_{h}$ and

$$
W_{h}(g)=\left\{v \in V_{h}: B_{h} v=g \text { in } Q_{h}^{\prime}\right\}
$$

where $g$ is restricted to $Q_{h}$.

In order to have the Galerkin approximation well defined we need to know that there exists a unique solution $\left(u_{h}, p_{h}\right) \in V_{h} \times Q_{h}$ of problem (5.18). In view of Theorem 5.5, this will be true if there exist $\alpha^{*}>0$ and $\beta^{*}>0$ such that 


$$
\begin{array}{ll}
\sup _{v \in W_{h}} \frac{a(u, v)}{\|v\|_{V}} \geq \alpha^{*}\|u\|_{V} \quad \forall u \in W_{h} \\
\sup _{u \in W_{h}} \frac{a(u, v)}{\|u\|_{V}} \geq \alpha^{*}\|v\|_{V} \quad \forall v \in W_{h}
\end{array}
$$

and,

$$
\sup _{v \in V_{h}} \frac{b(v, q)}{\|v\|_{V}} \geq \beta^{*}\|q\|_{Q} \quad \forall q \in Q_{h}
$$

In fact, (5.20) follows from (5.19) since $W_{h}$ is finite dimensional.

Now, we can prove the fundamental general error estimates due to Brezzi [13].

Theorem 5.6 If the forms $a$ and $b$ satisfy (5.19), (5.20) and (5.21), problem (5.18) has a unique solution and there exists a constant $C$, depending only on $\alpha^{*}, \beta^{*},\|a\|$ and $\|b\|$ such that the following estimates hold. In particular, if the constants $\alpha^{*}$ and $\beta^{*}$ are independent of $h$ then, $C$ is independent of $h$.

$$
\left\|u-u_{h}\right\|_{V}+\left\|p-p_{h}\right\|_{Q} \leq C\left\{\inf _{v \in V_{h}}\|u-v\|_{V}+\inf _{q \in Q_{h}}\|p-q\|_{Q}\right\}
$$

and, when $\operatorname{Ker} B_{h} \subset \operatorname{Ker} B$,

$$
\left\|u-u_{h}\right\|_{V} \leq C \inf _{v \in V_{h}}\|u-v\|_{V}
$$

Proof. From Theorem 5.5 we know that there exists a unique solution $\left(u_{h}, p_{h}\right) \in V_{h} \times Q_{h}$ of (5.18).

On the other hand, given $(v, q) \in V_{h} \times Q_{h}$, we have

$$
a\left(u_{h}-v, w\right)+b\left(w, p_{h}-q\right)=a(u-v, w)+b(w, p-q) \quad \forall w \in V_{h}
$$

and

$$
b\left(u_{h}-v, r\right)=b(u-v, r) \quad \forall r \in Q_{h} .
$$

Now, for fixed $(v, q)$, the right hand sides of (5.24) and (5.25)define linear functionals on $V_{h}$ and $Q_{h}$ which are continuous with norms bounded by

$$
\|a\|\|u-v\|_{V}+\|b\|\|p-q\|_{Q} \quad \text { and } \quad\|b\|\|u-v\|_{V}
$$


respectively. Then, it follows from Theorem 5.5 that, for any $(v, q) \in V_{h} \times Q_{h}$,

$$
\left\|u_{h}-v\right\|_{V}+\left\|p_{h}-q\right\|_{Q} \leq C\left\{\|u-v\|_{V}+\|p-q\|_{Q}\right\}
$$

and therefore (5.22) follows by the triangular inequality.

On the other hand, we know that $u_{h} \in W_{h}(g)$ is the solution of

$$
a\left(u_{h}, v\right)=\langle f, v\rangle \quad \forall v \in W_{h}
$$

and, since $W_{h} \subset W$, subtracting (5.26) from (5.4) we have,

$$
a\left(u-u_{h}, v\right)=0 \quad \forall v \in W_{h}
$$

Now, for $w \in W_{h}(g), u_{h}-w \in W_{h}$ and so from (5.19) and (5.27) we have

$$
\alpha^{*}\left\|u_{h}-w\right\|_{V} \leq \sup _{v \in W_{h}} \frac{a\left(u_{h}-w, v\right)}{\|v\|_{V}}=\sup _{v \in W_{h}} \frac{a(u-w, v)}{\|v\|_{V}} \leq\|a\|\|u-w\|_{V}
$$

and therefore,

$$
\left\|u-u_{h}\right\|_{V} \leq\left(1+\frac{\|a\|}{\alpha^{*}}\right) \inf _{w \in W_{h}(g)}\|u-w\|_{V}
$$

To conclude the proof we will see that, if (5.21) holds then,

$$
\inf _{w \in W_{h}(g)}\|u-w\|_{V} \leq\left(1+\frac{\|b\|}{\beta^{*}}\right) \inf _{v \in V_{h}}\|u-v\|_{V} .
$$

Given $v \in V_{h}$, from Lemma 5.2 we know that there exists a unique $z \in W_{h}^{\perp}$ such that

$$
b(z, q)=b(u-v, q) \quad \forall q \in Q_{h}
$$

and

$$
\|z\|_{V} \leq \frac{\|b\|}{\beta^{*}}\|u-v\|_{V}
$$

thus, $w=z+v \in V_{h}$ satisfies $B_{h} w=g$, that is, $w \in W_{h}(g)$. But

$$
\|u-w\|_{V} \leq\|u-v\|_{V}+\|z\|_{V} \leq\left(1+\frac{\|b\|}{\beta^{*}}\right)\|u-v\|_{V}
$$

and so (5.28) holds.

In the applications, a very useful criterion to check the inf-sup condition (5.21) is the following result due to Fortin [32]. 
Theorem 5.7 Assume that (5.7) holds. Then, the discrete inf-sup condition (5.21) holds with a constant $\beta^{*}>0$ independent of $h$, if and only if, there exists an operator

$$
\Pi_{h}: V \rightarrow V_{h}
$$

such that

$$
b\left(v-\Pi_{h} v, q\right)=0 \quad \forall v \in V, \forall q \in Q_{h}
$$

and,

$$
\left\|\Pi_{h} v\right\|_{V} \leq C\|v\|_{V} \quad \forall v \in V
$$

with a constant $C>0$ independent of $h$.

Proof. Assume that such an operator $\Pi_{h}$ exists. Then, from (5.29), (5.30) and (5.7) we have, for $q \in Q_{h}$,

$$
\beta\|q\|_{Q} \leq \sup _{v \in V} \frac{b(v, q)}{\|v\|_{V}}=\sup _{v \in V} \frac{b\left(\Pi_{h} v, q\right)}{\|v\|_{V}} \leq C \sup _{v \in V} \frac{b\left(\Pi_{h} v, q\right)}{\left\|\Pi_{h} v\right\|_{V}}
$$

and therefore, (5.21) holds with $\beta^{*}=\beta / C$.

Conversely, suppose that (5.21) holds with $\beta^{*}$ independent of $h$. Then, from (5.9) we know that for any $v \in V$ there exists a unique $v_{h} \in W_{h}^{\perp}$ such that

$$
b\left(v_{h}, q\right)=b(v, q) \quad \forall q \in Q_{h}
$$

and,

$$
\left\|v_{h}\right\|_{V} \leq \frac{\|b\|}{\beta^{*}}\|v\|_{V}
$$

Therefore, $\Pi_{h} v=v_{h}$ defines the required operator.

Remark 5.1 In practice, it is sometimes enough to show the existence of the operator $\Pi_{h}$ on a subspace $S \subset V$, where the exact solution belongs, verifying (5.29) and (5.30) for $v \in S$ and the norm on the right hand side of (5.30) replaced by a strongest norm (that of the space $S$ ). This is in some cases easier because the explicit construction of the operator $\Pi_{h}$ requires regularity assumptions which do not hold for a general function in $V$. For example, in the problem analyzed in the previous sections we have constructed this operator on a subspace of $V=H($ div,$\Omega)$ because the degrees of freedom defining the operator do not make sense in $H(d i v, T)$, indeed, we need more regularity for $\mathbf{v}$ (for example $\mathbf{v} \in H^{1}(T)^{n}$ ) in order to have the integral of the normal component of $\mathbf{v}$ against a polynomial on a face $F$ of $T$ well defined. It is possible to show the existence of $\Pi_{h}$ defined on $H($ div,$\Omega)$ satisfying (5.29) and (5.30) (see [32, 46]). However, as we have seen, this is not really necessary to obtain optimal error estimates. 


\section{References}

[1] Acosta, G.\& Durán, R. G, The maximum angle condition for mixed and non conforming elements : Application to the Stokes equations, SIAM J. Numer. Anal. 37, 18-36, 2000.

[2] Acosta, G., Durán, R. G \& Muschietti,M. A., Solutions of the divergence operator on John domains, Advances in Mathematics 206, 373-401, 2006.

[3] Ainsworth, M., Robust a posteriori error estimation for nonconforming finite element approxiamations, SIAM J. Numer. Anal. 42, 23202341, 2005.

[4] Alonso, A., Error estimator for a mixed method, Numer. Math. 74, 385-395, 1996.

[5] Arnold, D. N., Boffi, D. \& Falk, R. S., Quadrilateral H(div) finite elements, SIAM J. Numer. Anal. 42, 2429-2451, 2005.

[6] Arnold, D. N \& Brezzi, F.. Mixed and nonconforming finite element methods implementation, postprocessing and error estimates, R.A.I.R.O., Modél. Math. Anal. Numer. 19, 7-32, 1985.

[7] Arnold, D. N, Scott, L. R \& Vogelius, M, Regular inversion of the divergence operator with Dirichlet boundary conditions on a polygon, Ann. Scuola Norm. Sup. Pisa Cl. Sci-Serie IV, XV, 169-192, 1988 .

[8] Babuska, I., Error bounds for finite element method, Numer. Math. 16, 322-333, 1971.

[9] Babuska, I., The finite element method with lagrangian multipliers, Numer. Math., 20, 179-192, 1973.

[10] Bermúdez, A. , Gamallo, P. , Nogueiras, M. R \& Rodríguez, R. Approximation of a structural acoustic vibration problem by hexhaedral finite elements, IMA J. Numer. Anal. 26, 391-421, 2006.

[11] Bramble, J.H.\& Xu, J.M., Local post-processing technique for improving the accuracy in mixed finite element approximations, SIAM J. Numer. Anal. 26, 1267-1275, 1989. 
[12] Brenner, S.\& Scott, L. R. The Mathematical Analysis of Finite Element Methods, Springer Verlag, 1994.

[13] Brezzi, F., On the existence, uniqueness and approximation of saddle point problems arising from lagrangian multipliers, R.A.I.R.O. Anal. Numer. 8, 129-151, 1974.

[14] Brezzi, F., Douglas, J., Durán, R.\& Fortin, M., Mixed finite elements for second order elliptic problems in three variables, Numer. Math. 51, 237-250, 1987.

[15] Brezzi, F., Douglas, J., Fortin, M.\& Marini, L. D. Efficient rectangular mixed finite elements in two and three space variables, Math. Model. Numer. Anal. 21, 581-604, 1987.

[16] Brezzi, F., Douglas, J.\& Marini, L. D.. Two families of mixed finite elements for second order elliptic problems, Numer. Math. 47, 217-235, 1985.

[17] Brezzi, F.\& Fortin, M., Mixed and Hybrid Finite Element Methods, Springer Verlag, 1991.

[18] Carstensen, C., A posteriori error estimate for the mixed finite element method, Math. Comp. 66, 465-476, 1997.

[19] Ciarlet, P. G.,, The Finite Element Method for Elliptic Problems, North Holland, 1978.

[20] Ciarlet, P. G., Mathematical Elasticity, Volume 1. ThreeDimensional Elasticity, North Holland, 1988.

[21] Clément, P., Approximation by finite element function using local regularization, RAIRO R-2, 77-84, 1975.

[22] Crouzeix, M.,\& Raviart, P. A., Conforming and non-conforming finite element methods for solving the stationary Stokes equations, R.A.I.R.O. Anal. Numer. 7, 33-76, 1973.

[23] Dari, E., Durán, R. G., Padra, C.\& Vampa, V., A posteriori error estimators for nonconforming finite element methods, Math. Model. Numer. Anal. 30, 385-400, 1996.

[24] Douglas, J.\& Roberts, J. E. Global estimates for mixed methods for second order elliptic equations, Math. Comp. 44, 39-52, 1985. 
[25] Dupont, T.\& Scott, L. R, Polynomial approximation of functions in Sobolev spaces, Math. Comp. 34, 441-463, 1980.

[26] Durán, R. G., On polynomial Approximation in Sobolev Spaces, SIAM J. Numer. Anal. 20, 985-988, 1983.

[27] Durán, R. G, Error Analysis in $L^{p}$ for Mixed Finite Element Methods for Linear and quasilinear elliptic problems, R.A.I.R.O. Anal. Numér $22,371-387,1988$.

[28] Durán, R. G., Error estimates for anisotropic finite elements and applications, Proceedings of the International Congress of Mathematicians, 1181-12002006.

[29] Durán, R. G.\& Lombardi, A. L., Error estimates for the RaviartThomas interpolation under the maximum angle condition, preprint, http://mate.dm.uba.ar/ rduran/papers/dl3.pdf .

[30] Durán, R. G. \& Muschietti, M. A., An explicit right inverse of the divergence operator which is continuous in weighted norms, Studia Math. 148, 207-219, 2001.

[31] Falk, R. S., Osborn, J., Error estimates for mixed methods, R.A.I.R.O. Anal. Numer. 4, 249-277, 1980.

[32] Fortin, M., An analysis of the convergence of mixed finite element methods, R.A.I.R.O. Anal. Numer. 11, 341-354, 1977.

[33] Gagliardo, E., Caratterizzazioni delle tracce sulla frontiera relative ad alcune classi di funzioni in $n$ variabili, Rend. Sem. Mat. Univ. Padova 27, 284-305, 1957.

[34] Gastaldi, L. \& Nochetto, R. H., Optimal $L^{\infty}$ - error estimates for nonconforming and mixed finite element methods of lowest order, Numer. Math. 50, 587-611, 1987.

[35] Gastaldi, L. \& Nochetto, R. H., On $L^{\infty}$ - accuracy of mixed finite element methods for second order elliptic problems, Mat. Aplic. Comp. 7, 13-39, 1988.

[36] Gilbarg, D.\& Trudinger, N. S., Elliptic Partial Differential Equations of Second Order, Springer Verlag, 1983. 
[37] Girault, V. \& Raviart, P. A. Finite Element Methods for NavierStokes Equations, Berlin, Springer-Verlag, 1986.

[38] Grisvard, P., Elliptic Problems in Nonsmooth Domain, Pitman, Boston, 1985.

[39] Lovadina, C.\& Stenberg, R., Energy norm a posteriori error estimates for mixed finite element methods, Math. Comp. 75, 1659-1674, 2006.

[40] Marini, L. D., An inexpensive method for the evaluation of the solution of the lowest order Raviart-Thomas mixed method, SIAM J. Numer. Anal. 22, 493-496, 1985.

[41] Nedelec, J. C., Mixed finite elements in $\mathbb{R}^{3}$, Numer. Math. 35, 315$341,1980$.

[42] Nedelec, J. C., A new family of mixed finite elements in $\mathbb{R}^{3}$, Numer. Math. 50, 57-81, 1986.

[43] Payne, L. E., Weinberger, H. F., An optimal Poincaré inequality for convex domains, Arch. Rat. Mech. Anal. 5, 286-292, 1960.

[44] Raviart, P. A., Thomas, J. M., A mixed finite element method for second order elliptic problems, Mathematical Aspects of the Finite Element Method, (I. Galligani, E. Magenes, eds.), Lectures Notes in Math. 606, Springer Verlag, 1977.

[45] Roberts, J. E., Thomas, J. M., Mixed and Hybrid Methods, in Handbook of Numerical Analysis, (P.G. Ciarlet and J.L. Lions,eds.), Vol.II, Finite Element Methods (Part 1), North Holland, 1989.

[46] SchöBerl, J., Commuting quasi-interpolation operators for mixed finite elements, Preprint ISC-01-10-MATH, Texas A\&M University, 2001.

[47] Scott, L. R., Zhang, S., Finite element interpolation of non-smooth functions satisfying boundary conditions, Math. Comp. 54, 483-493, 1990 .

[48] Stenberg, R.,, Postprocessing schemes for some mixed finite elements, RAIRO, Model. Math. Anal. Numer. 25, 151-167, 1991. 
[49] Thomas, J. M. Sur l'Analyse Numérique des Méthodes d'Éléments Finis Hybrides et Mixtes. Thèse de Doctorat d'Etat, Université Pierre et Marie Curie, Paris, 1977.

[50] Verfürth, R., A posteriori error estimators for the Stokes equations, Numer. Math. 55, 309-325, 1989.

[51] Verfürth, R., A note on polynomial approximation in Sobolev spaces, RAIRO M2AN 33, 715-719, 1999.

[52] R. Verfürth, A Review of A Posteriori Error Estimation and Adaptive Mesh-Refinement Techniques, Wiley \& Teubner, 1996. 